\title{
Stimuli-controlled fluid control and microvehicle movement in microfluidic channels
}

\author{
Aishling Dunne ${ }^{\dagger}$, Wayne Francis ${ }^{\dagger}$, Colm Delaney, Larisa Florea* and Dermot Diamond
}

$\dagger$ Both authors contributed equally to this work.

Insight Centre for Data Analytics, National Centre for Sensors Research, School of Chemical Sciences, Dublin City University, Dublin, Ireland. E-Mails: wayne.francis2@mail.dcu.ie; aishling.dunne58@mail.dcu.ie; colm.delaney@dcu.ie.

* Author to whom correspondence should be addressed; E-Mail: larisa.florea@dcu.ie; Tel.: +3531700 6009; Fax: +35317008021.

\begin{abstract}
Integration of stimuli-responsive materials into microfluidic systems provides a means to locally manipulate flow at the microscale, in a non-invasive manner, while also reducing system complexity. In recent years, several modes of stimulation have been applied, including electrical, magnetic, light and temperature, among others. To achieve remote control of flow in microfluidics using external stimulation, two main approaches have emerged in the recent years:

1. Control of flow through stimuli-induced actuation of microfluidic components (valves, pumps, mixers, flow sorters), most often fabricated from soft polymeric materials;

2. Stimuli-controlled manipulation of discrete micrometer-sized "vehicles" (droplets, beads, Janus particles, etc.) through localized induced changes in wettability or surface tension.
\end{abstract}

The focus of this chapter will be to identify and compare the similarities and underlying mechanisms employed in the current state of the art research in stimuli-controlled fluid control and micro-vehicle movement fields. It will also endeavor to propose possible directions for the evolution of these areas of research.

Keywords: microfluidics; stimuli-responsive materials; flow control; polymer actuators.

\section{Introduction}

Since the onset of research into microfluidics during the 1990s, there has been much progress made in the generation and control of flow in micrometre-sized devices.[1,2] The aim of these tiny devices is to perform complex biological and chemical tasks on a single chip.[3] Advantages of this approach include a significant reduction in reactant volume (down to microlitres or even picolitres), parallel processing of multiple analytes, portability,[4] multistage automation,[5] single cell sampling[6] and manipulation.[7] Therefore, the potential for microfluidic devices is tremendous. Nevertheless, the microfluidics field is still very much in its infancy and a number of areas are constantly under heavy investigation, including micro-fabrication, component integration, device generalisation and fluid flow and control.[8] Despite considerable advances in recent years, fluid handling on microfluidic chips still relies, in many cases, on macroscopic external control boxes containing power supplies, high power sources, sensing elements and complicated control systems based on external computers and/or electronics.[9] While there are still many challenges facing this field, there have been a number of revolutionary approaches, one of the most exciting of which is the incorporation of stimuli-responsive actuators within the fluidics, to achieve integrated fluid handling on-chip. This approach can result in flow control,[10, 11] mixing,[12] flow sorting,[13] pumping[14] and even sensing components fully integrated into the microfluidics chip [15]. Recent exciting developments in stimuli-responsive materials, and particularly stimuli responsive polymers and surfaces make microfluidics a great platform for demonstrating the capabilities and highlighting the functionality of these materials.[16, 
17] Indeed, the microfluidic platform generally enhances the functionality of smart materials by offering faster kinetics and shorter reaction times due to increased surface to volume ratios; improved interfacial and chemical interactions; dominant surface tension and capillarity effects and efficient absorption of electro-magnetic radiation.

To date, the main approaches to achieve flow control in microfluidic devices using stimuliresponsive materials, include:

1. The indirect approach, where flow control is realized through stimuli-induced actuation of microfluidic components (valves, pumps, mixers, flow sorters), most often fabricated from soft polymeric materials or polymer thin films;

2. The direct approach, where discrete micrometre-sized droplets are externally manipulated through stimuli-induced changes in wettability or surface tension.

This chapter will focus on these particular approaches for stimuli-controlled fluid control and microvehicle movement, with the motivation of identifying and comparing current state of the art methodologies and offering the reader an up-to-date view of this exciting research area.

\section{Control of flow through stimuli-induced actuation of microfluidic components}

The most commonly used stimuli-responsive actuators in microfluidic devices are based on multilayer polymer films or soft polymer hydrogels. Although the actuation mechanism of these two classes is considerably different, they offer similar functionality to microfluidic devices through the generation of valves, pumps, flow sorters, filters and mixers[18].

\subsection{Multilayer Polymer Films}

Thin film actuators are based on a bilayer principle, in which two materials respond differently to a chosen stimulus[19]. Such bilayers are commonly comprised of a metal and conducting polymer, such as a gold/polypyrrole couple, where metal cations are inserted into the polymer upon reduction and removed when the polymer is oxidised $[20,21]$. Depending on the doping regime in the conducting polymer, a positive bias usually generates the oxidised state, leading to an increase in volume. Application of a negative bias can then result in actuation of the material. The oxidation rate of the polymer can be controlled by variation of the applied voltage. These properties result in a wide variety of applications for conductive polymer actuators, such as artificial muscles[22], biomedical devices[23], microfluidics[24] and novel actuators[25-27]. A pertinent example by Smela et al.[21] demonstrates an electrochemically driven conducting polymer bilayer, based on a doped gold/polypyrrole bilayer fabricated via reactive ion etching (RIE) (shown in Figure 1), which can be used as a hinge to move silicon plates.

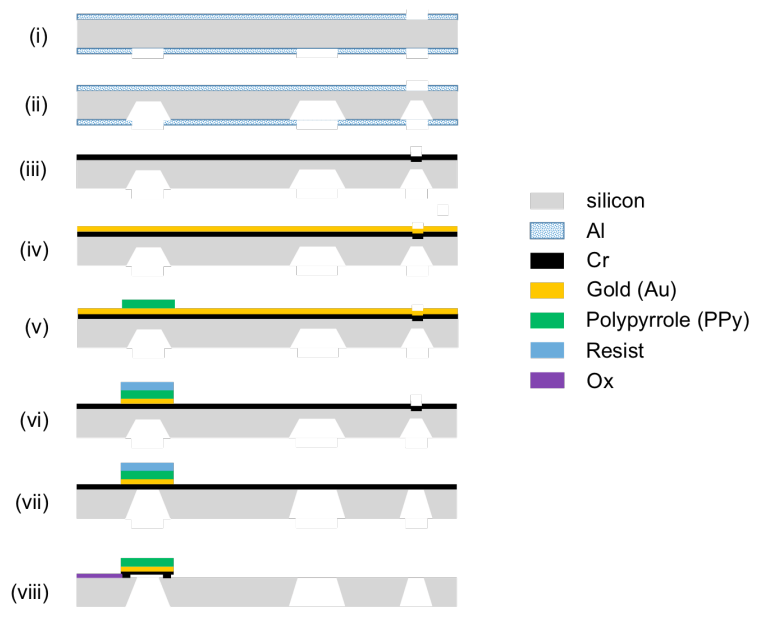


Figure 1. Manufacture schematic: (i) patterned etch mask applied for RIE, (ii) RIE etch to define SI plates, (iii) deposit of $\mathrm{SiO}_{2}$, (iv) deposit of $\mathrm{Cr}$ and $\mathrm{Au}$, (v) deposit and pattern of PPy, (vi) pattern of Cr and Au, (vii) finished etching, (viii) free plates and hinge by etching $\mathrm{SiO}_{2}$ (Adapted from Smela et al. [21]).

The actuation speeds and lifetime of these Au/PPy-DBS electroactive bilayer hinges, which operate in a voltage range of 0 to $-1 \mathrm{~V}$, are extremely reliant on the thickness of the bilayer, and hampered by the possibility of delamination. These disadvantages were significantly outweighed by the high strength, low required voltage and the control of actuation observed (Figure 2). Such actuator hinges are envisioned for lab-on-a-chip applications where they can be used to control sealable lids over silicon etched cavities to capture cells.

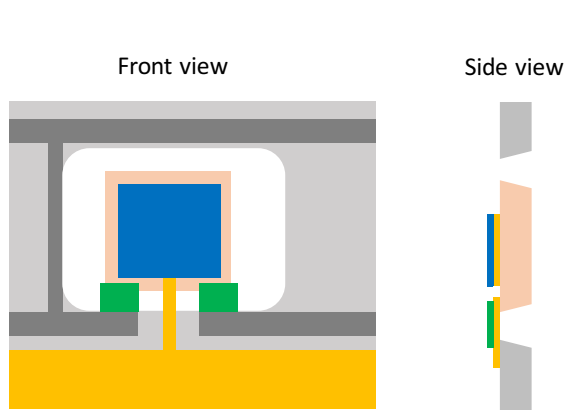

(a)
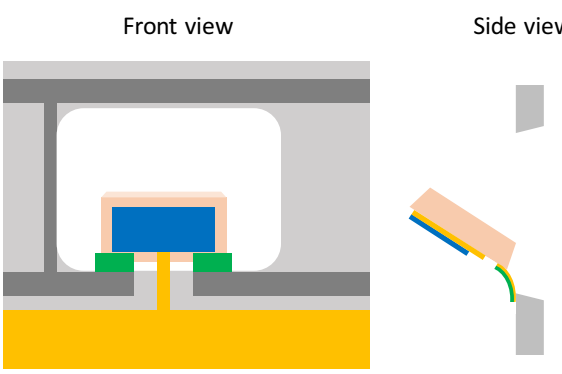

(b)

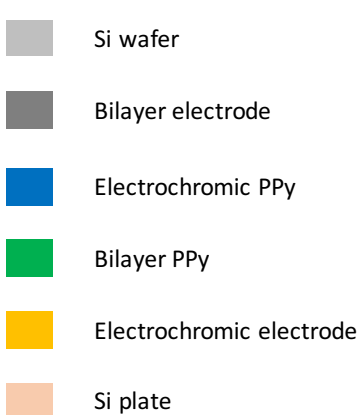

Si plate

Figure 2. Schematic image of Si plate rotating on PPy/gold hinges; a) Bilayer PPy is reduced, plate lies flat. B) Bilayer PPy is partly oxidized, plate rotates out of the plane. (the electrochromic PPy is oxidised in both) (Adapted from E. Smela [28]).

The scope of this research has been further developed with alternative materials to broaden potential applications in microfluidic devices, in the work undertaken by Tanaka et al.[29]. They report an electroactive polymer-based micro-stop valve occupying a small space on a microchip. This valve was achieved by taking advantage of the controlled deformation of the electroactive polymer by an applied voltage. The valve, created by positioning the electroactive polymer film membrane between two $5 \mu \mathrm{m}$ thick soft electrode sheets, was placed on top of a $500 \mu \mathrm{m}$ glass microchannel using a silicon rubber diaphragm (Figure 3). The flow in the microchannel produced by constant pressure from a microfluidic controller (under $4.0 \mathrm{kPa}$ ) was completely stopped within $1 \mathrm{~s}$ by applying an electric field of $60 \mathrm{~V} / \mu \mathrm{m}$.

Valve open

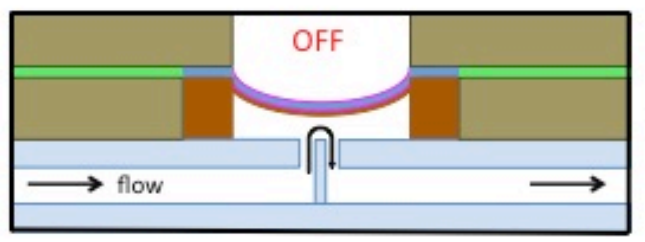

Acrylic resin

Wire

Soft electrode
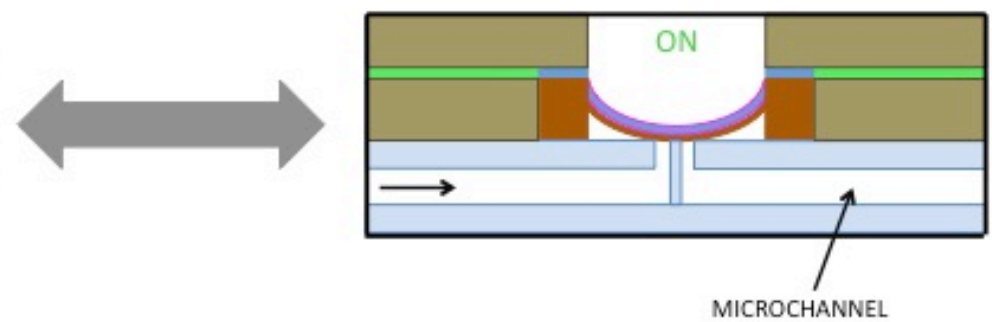

Electroactive polymer

Elastomer

Glass 
Figure 3. Design and working principles of the stop valve incorporated into the microfluidic chip. (Adapted from Y. Tanaka et al.[29]).

Another microfluidic actuator has been developed using a hybrid device consisting of an electroactive polymer within an electro-adaptive microfluidic (EAM) system. Recent investigations resulted in an EAM device which consisted of a five layer acrylic dielectric elastomer ( $\mathrm{VHB}^{\mathrm{TM}}$ tape), single walled carbon nanotubes (SWCNT) and a $60 \mu \mathrm{m}$ casting layer of PDMS to protect the electroactive polymer. A microfluidic channel made of PDMS, as seen in Figure 4, was attached to the electroactive polymer by oxygen plasma bonding. This multi-layered actuator allowed for modulation of the shape and dimensions of a microfluidic channel with the applied bias voltage, and a consequence permitted fluidic resistance alteration. This type of hybrid device can combat clogging issues in microfluidic systems, used for self-clearing channels, or provide design flexibility via tunable channel geometries [30].

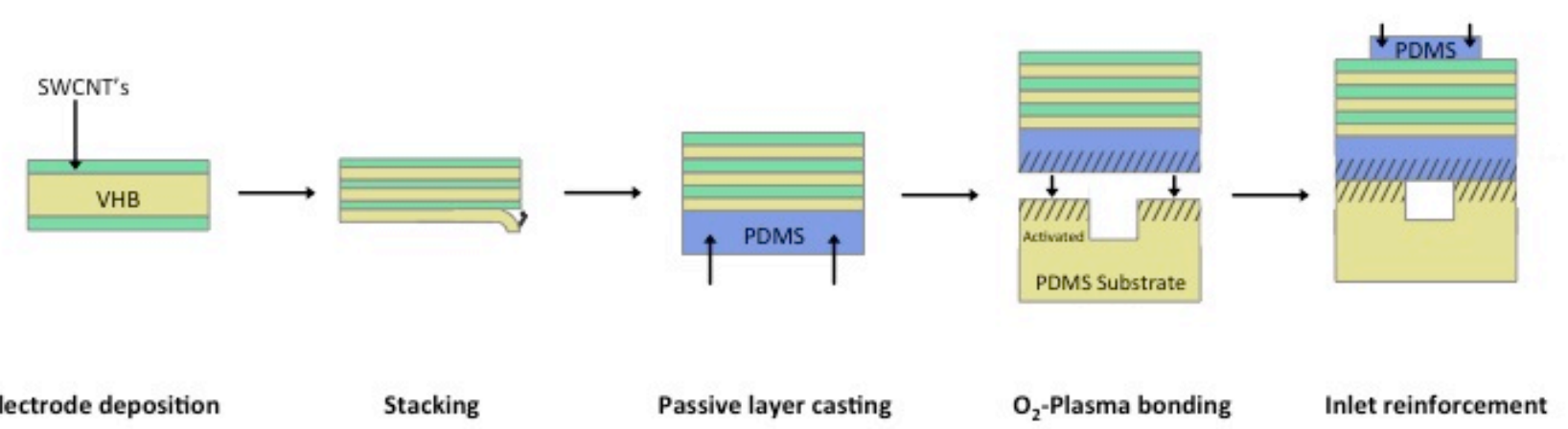

Figure 4. Overview of the fabrication steps involved for the electro-adaptive microfluidic system, including electrode deposition onto the VHB acrylic elastomer, stacking of these actuators, casting of the PDMS passive layer, activating the PDMS substrate and passive layer via air plasma followed by bonding them, and reinforcing the inlet by casting a short PDMS strip over the inlet. . (Adapted from C. Murray et al. [30]).

\subsection{Soft actuators - Hydrogels}

Hydrogels are an alternative to thin films, considerably more exploited in microfluidics, mainly due to their ease of fabrication and integration and also to their compatibility with aqueous media[13, 18, 31, 32]. Due to the hydrophilic character of hydrogels, strong associative interactions between polymer chains and water molecules occur, resulting in a high degree of water absorption (up to $95 \%$ of total mass) $[13,33]$. As the swelling of the hydrogel is a diffusion-controlled process, the microfluidic systems provide an ideal platform to demonstrate hydrogel functionality, as the micro/nanoscale dimensions considerably reduce water the diffusion pathlengths, thereby improving actuation kinetics that in some cases can reduce hydrogel response time from hours to seconds[34, 35].

The capability of a hydrogel to absorb water can be altered through the introduction of competitive hydrophobic segments inside the hydrophilic network. These hydrophobic segments facilitate polymerpolymer interactions, causing the polymer to collapse and expel water molecules. Thus, the capacity of a hydrogel to absorb water relies on competing polymer-polymer and polymer-water interactions which are strongly dependent on the ratio of hydrophilic to hydrophobic segments present in the macromolecular network. When the hydrophilic interactions dominate, the polymer interacts with the surrounding water molecules through hydrogen bonds and the hydrogel swells due to water ingress (Figure 5(a)). Conversely, when hydrophobic interactions dominate, the polymer-polymer interactions are increased, causing the hydrogel to shrink (Figure 5(b)). Therefore, by varying the ratio between the hydrophobic/hydrophilic segments inside the macromolecular network, the shrinking/swelling behaviour of the hydrogel can be easily controlled (Figure 5). 
Conveniently, the hydrophilic/hydrophobic character of many polymers and chemical groups can be altered by applying different stimuli, such as temperature, $\mathrm{pH}$, electric fields or light, among others[36, 37].
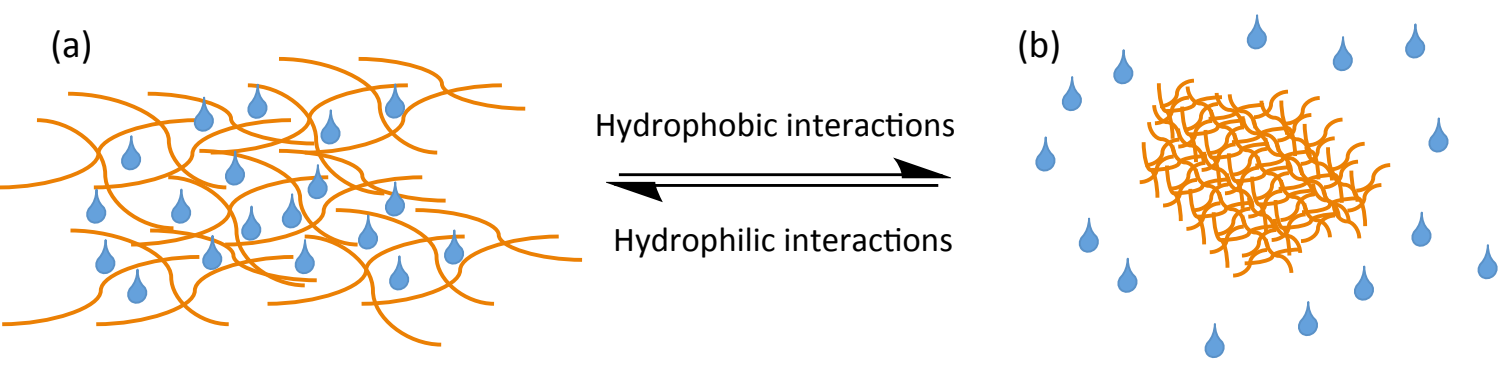

$$
\checkmark=\text { Water/solution } S=\text { polymer chains }
$$

Figure 5. Scheme showing the reversible shrinking and swelling of a hydrogel based on dominant hydrophilic (left) or hydrophobic (right) interactions, respectively.

\subsection{1 pH-induced actuation}

$\mathrm{pH}$ responsive hydrogel systems have multiple potential applications in the biomedical[38], environmental[39] and drug delivery fields[40, 41]. $\mathrm{pH}$ responsive hydrogels change volume when exposed solutions of differing $\mathrm{pH}$. Such polymeric materials are generally composed of monomeric units which carry an acidic or basic group in their structure; such as carboxyl or amine groups. The effective $\mathrm{pH}$ is determined by the $\mathrm{pK}_{\mathrm{a}}$ of the $\mathrm{pH}$ responsive sensing material within the hydrogel. Around this $\mathrm{pH}$, the responsive material will ionize through protonation/deprotonation, resulting in an alteration of charge on the polymer chain and thus causing a change in the balance of polymer/water polymer/polymer interactions thereby modulating the physical size of the hydrogel. A variety of $\mathrm{pH}$ responsive hydrogels have been developed to date, based on maleic anhydride (MA), methacrylic acid (MAA), N,N-dimethylaminoethyl methacrylate (DMAEMA) and acrylic acid (AA), among others $[42,43]$.

Beebe et al. [13] found that by clever design, a dual hydrogel system can simultaneously sense a chemical environment change in one channel and control the flow in a separate channel. In this work the hydrogels were composed of poly(2-hydroxyethyl methacrylate-co-acrylic acid) (p(HEMA-AA)) and poly(2-hydroxyethyl methacrylate-co-(dimethylamino)ethyl methacrylate) (p(-HEMA(dimethylamino)ethyl methacrylate)) and were used to control the direction of the flow based on the $\mathrm{pH}$ of the fluid passing inside the channel (Figure 6). At $\mathrm{pH} \mathrm{7.8,} \mathrm{the} \mathrm{p}$ (HEMA-(dimethylamino)ethyl methacrylate) hydrogel on the right expands to the walls of the channel constricting fluid flow while the hydrogel on the left is contracted resulting in the flow being directed down the left channel. Oppositely, at a $\mathrm{pH}$ of 4.7 , the flow is directed down the right branch as the p(HEMA-AA) hydrogel on the left has expanded and the hydrogel on the right has contracted. When the $\mathrm{pH}$ of the solution is $\sim 6.7$ both hydrogels expand blocking the channels and preventing any fluid flow. This work resulted in the successful demonstration of a "self-regulated flow sorter".

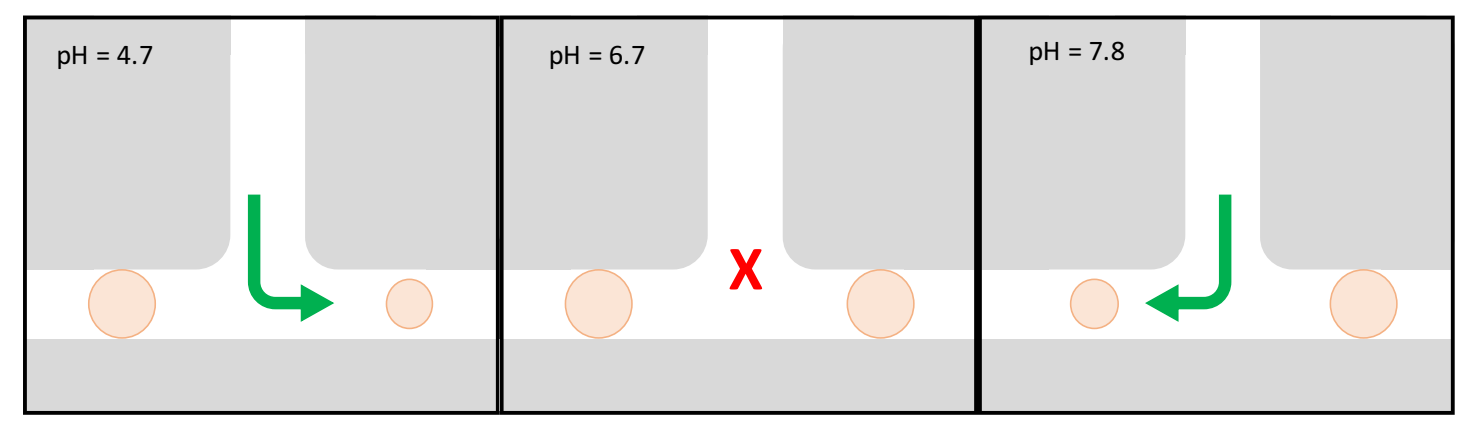


Figure 6. A microfluidic device demonstrating $p H$ sensitive hydrogels working as a pH sorter to control fluid flow. (Adapted from Beebe D. et al. [13]).

In the same study a second microfluidic system was created, using the same $\mathrm{pH}$-responsive materials (acrylic acid, 2-hydroxyethyl methacrylate and ethylene glycol dimethacrylate) to work as a shut off valve to control flow. The shut off valve design was composed of an upper and lower section, separated by a conformable membrane, with the hydrogel occupying the upper area and an aqueous solution occupying the lower area. When the hydrogel was exposed to solutions of varied $\mathrm{pH}$, expansion or contraction was observed. The hydrogel expansion resulted in the application of sufficient pressure being placed on the deformable membrane to cause it to close the channel below, thus blocking the flow (Figure 7).

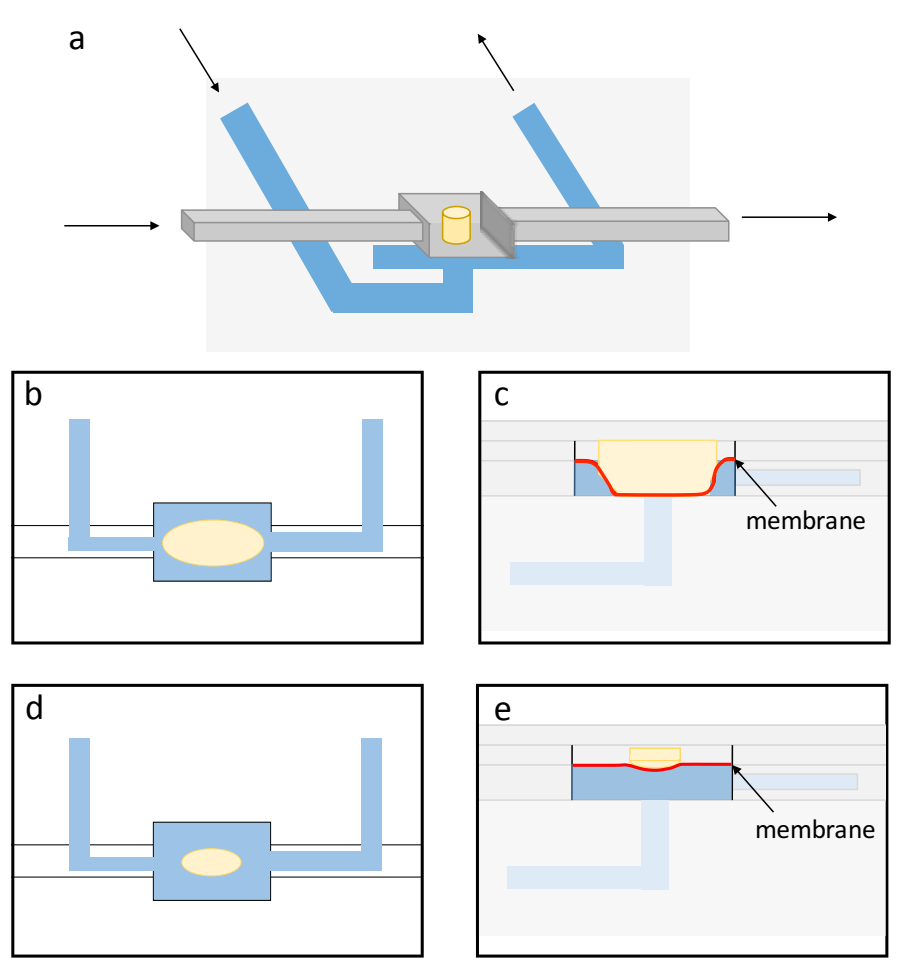

Figure 7. Schematic illustration and working mechanism of the shut-off valve; a) Diagram of the shut-off valve; b), c) top and side view, respectively, showing hydrogel expansion, deforming the membrane and blocking flow in the adjacent channel; d), e) top and side view, respectively, showing hydrogel contraction, causing the membrane to return to the initial position and allowing fluid to flow. (Adapted from Beebe D. et al. [13]).

\subsubsection{Thermo-induced actuation}

Thermo-responsive materials have greatly impacted research into actuating materials with many possible applications such as drug delivery[40, 43, 44], chromatography techniques[45], biomedicine[46-48] being explored.

The thermo-response arises from a shift in the hydration forces which occur between the polymer chains and the aqueous medium, at temperatures close to the lower critical solution temperature (LCST) of a polymer[49]. At temperatures below the LCST the dominant interactions taking place are hydrogen bonding between the hydrophilic sections of the polymer chain and water. This results in water uptake and the polymer chains are elongated with negligible internal stresses. When the polymer is in a temperature environment above the LCST, hydrophobic polymer-polymer interactions become dominant and hydrogen bonding and hydrophilic interactions weaken, resulting in aggregation of the linear chains into a globular form, due to expulsion of water with a concomitant decrease in the physical size of the hydrogel [50]. An important consideration for a thermo-response is the presence of 
both hydrophilic (i.e. amide, carboxyl etc.) and hydrophobic (i.e. alkyl, aryl etc.) groups in the polymer structure. Some of the most studied thermo-responsive materials include poly( $N$-isopropylacrylamide) (p(NIPAAm)) [43, 48, 50], and copolymers such as poly(2-(2-methoxyethoxy)ethyl methacrylate-cooligo(ethylene glycol)methacrylate)[51].

pNIPAAm hydrogels exhibit a negative volume change in response to increase in temperature, i.e., the pNIPAAm hydrogels shrink as the temperature increases above the LCST associated with the volume phase transition. Below the LCST $\left(32^{\circ} \mathrm{C}\right)$, the pNIPAAm polymer chains are soluble in water. Above the LCST, they become increasingly hydrophobic and insoluble, leading to precipitation. The thermal transition of pNIPAAm hydrogels is generally considered to be a competitive result of the hydrophobic interaction of pendant isopropyl groups and polymer segments (polymer-polymer interactions) and the hydrogen bonding association between amide groups and water molecules (polymer-water interactions).

pNIPAAm gels are being increasingly used in microfluidic systems as microscale valves. Two possible valve systems containing pNIPAAm gels have been produced by Wang et al. [52] which require no mechanical parts and exhibit minimal dead volume. The first example shows gel actuators which were fabricated outside the channel and compressed into a $1.2 \mathrm{~mm}$ diameter Teflon ${ }^{\mathrm{tm}}$ tube (Figure 8a).

Following this, the Teflon ${ }^{\mathrm{tm}}$ tube was placed on a heater $\left(58^{\circ} \mathrm{C}\right)$, causing valve contraction, thereby opening the tube and allowing for the flow of fluid. The closing time of the hydrogel valve was about $4.5 \mathrm{~s}$, and it was found to be independent of the plug's length as it relies on the local swelling of the upstream end of the gel plug. Average opening times were $5 \mathrm{~s}$ and $12 \mathrm{~s}$, when the hydrogel length was $300 \mu \mathrm{m}$ and $1500 \mu \mathrm{m}$, respectively. This size dependency is due to the fact that the entire length of the gel plug must de-swell, and the excess water must be expelled through the gel's length, before the fluid can flow through the tube. A second design was fabricated (Figure 8b), in order to improve valve performance and tolerance.

(a)
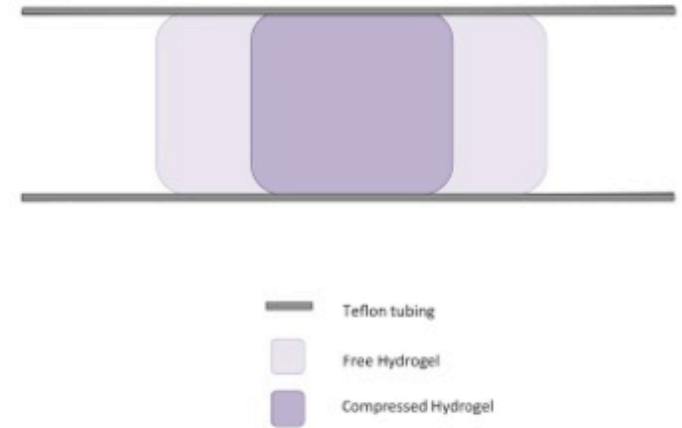

(b)

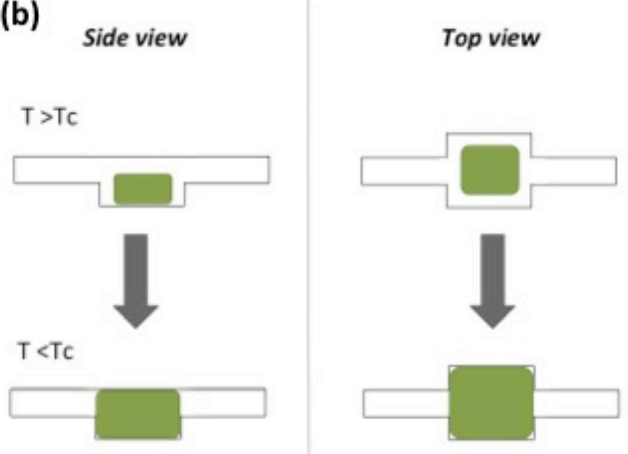

Figure 8. Schematic illustration of the pNIPAAm thermo-responsive hydrogel valve; (a) shows the hydrogel plug confined in the Teflon tube under free conditions and when compressed by two stainless steel rods that fit into the Teflon tube; (b) shows the side and top views of the valve when incorporated into a polycarbonate microfluidic devices at temperatures above (valve open) and below (valve closed) the critical temperature (Tc). Adapted from J. Wang et al. [52]

One important advance in this field is the in-situ polymerisation of hydrogels within microfluidic devices. In the work by Giger et al. [33], a p(NIPAAm) based polymer matrix was photopolymerised using UV-light within an injection-molded channel. The thermal response was investigated for both on and off-chip actuation. Off-chip actuation involved the manual movement of the device on/off an aluminium hot plate set at $45{ }^{\circ} \mathrm{C}$. On-chip actuation was made possible through the use of "on-chip" micro-heaters. In both cases, when the heat supplied to the systems reached a temperature at or above the LCST of p(NIPAAm), the polymer matrix contracted in volume, and the off-chip and on-chip opening times were $\sim 3 \mathrm{~s}$. Off-chip reclosing of the channel took $\sim 20 \mathrm{~s}$ compared to $\sim 5 \mathrm{~s}$ for on-chip reclosing. The results demonstrate that incorporation of micro-heaters into the 
microfluidic device can enable fast actuation and successful repeatability when compared to the manual off-chip method [33]. Recently this ability to use thermo-responsive hydrogels in autonomous microfluidic systems with little or no human intervention has been further improved through the work of Agarwal et al., [53] through their work on "autonomously-triggered on-chip microfluidic cooling devices". They integrated a thermally-responsive polymer material at the axle of a nickel impeller with the purpose of controlling the impeller rotation autonomously. This study employed p(NIPAAm) as the thermally-sensitive actuation material in a microfluidic system which comprised microchannels, an on-chip cooling reservoir, an external heater and an external magnetic stirrer (Figure 9). At temperatures above the LCST, the polymer material contracted, enabling the rotation of the impeller and allowing fluid flow from the reservoir to cool the system. The opposite phenomenon was observed below the LCST, when expansion of the hydrogel prevented the Ni impeller from rotating. Integration of this thermo-responsive hydrogel into the aforementioned system as an actuator therefore provides the basis of autonomously-triggered on-chip microfluidic cooling device that could have applicability in a number of scenarios where on-chip temperature control and regulation is necessary.

(a)

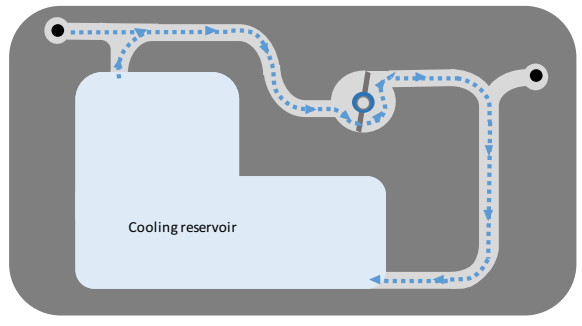

(b)

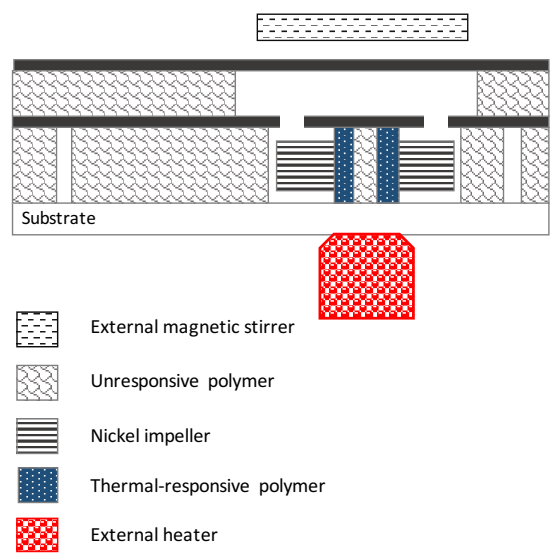

Figure 9. (a) Schematic illustration of the autonomously-triggered on-chip microfluidic cooling device. The dashed blue line and arrows show the pathway of the fluid as it is pumped and recirculated through the microchannels; (b) Cross-section of the device showing the device components; when the temperature of the heater is $32{ }^{\circ} \mathrm{C}$, the hydrogel contracts and the $\mathrm{Ni}$ impeller rotates, pumping and recirculating cool water. Oppositely, when local temperatures drop below $32{ }^{\circ} \mathrm{C}$, the hydrogel expands stopping the Ni impeller from rotating. (Adapted from Agarwal A. K. [53]).

\subsubsection{Photo-induced actuation}

Light-driven responsiveness in polymers is normally achieved through the inclusion of a photochromic molecule, either by non-covalent doping or covalent attachment. When the photochromic unit is exposed to light of specific wavelengths[54-58] it exhibits an isomerisation change between two or more isomers that can have dramatically different properties (e.g. polarity, hydrophilic/hydrophobic character, geometry, charge density), that in turn can affect the properties of the surrounding polymeric matrix. Many photochromic molecules have been studied, but three of the best-known are spirobenzopyrans (SP) [54-56, 59, 60], azobenzenes[61, 62], and diarylethenes[63], all of which show reversible photochromic behaviour, transforming from one isomer to another when exposed to 
different wavelengths of light. Much research has been carried out into optimising the photo-response of these materials when incorporated into hydrogel matrices, for exploitation in microfluidic systems as valves, pumps, mixers and alternating the topography of surfaces[64-67]. In addition to the development of the materials themselves (e.g. for optimisation of the photo-response kinetics), various polymerisation strategies have been investigated to enable the generation of precise micro-structures. Amongst them, the use of micropatterened light irradiation[68] enables specific hydrogels of different shapes and sizes to be polymerised using pre-designed photo-masks.

Sugiura et al. [69] first reported the use of photo-responsive hydrogels based on pNIPAAm as microfluidic integrated valves in acidic environments. The microfluidic platform presented was made of PDMS composed of three adjacent micro-channels, each with a micro-pillar around which the photo-responsive hydrogel was polymerized (Figure 10). Using three micro-channels in parallel, enabled individual valve actuation without the interference of adjacent channels to be demonstrated. The polymer material was p(NIPAAm) functionalised with a spirobenzopyran (SP) chromophore, photo-polymerised in situ around the micro-pillars to form micro-valves (Figure 10). In the dark, when SP-functionalised $\mathrm{p}$ (NIPAAm) is exposed to acidic conditions (e.g. $\mathrm{pH}$ 2-3), the following interactions occur: (1) the less hydrophilic colourless SP will transform to the more hydrophilic protonated, yellow coloured merocyanine $\left(\mathrm{MC}-\mathrm{H}^{+}\right)$; (2) this triggers a conversion of the gel to a more hydrophilic configuration in which polymer-water interactions dominate; (3) the pNIPAAm-co-SP hydrogel expands as more water is drawn in through diffusion. When exposed to visible light, the hydrogel material contracts, as the $\mathrm{MC}-\mathrm{H}^{+}$isomer reverts to the $\mathrm{SP}$ form, triggering precipitation of the pNIPAAm chains and expulsion of water from the gel.

To enable reversible actuation of this material, the hydrogel required an acidic environment $(0.05 \mathrm{mM} \mathrm{HCl})$. Each valve was tested separately and a reduction of $52 \%$ in diameter was obtained within $18-32 \mathrm{~s}$ of light irradiation. After a total of $120 \mathrm{~s}$, the gel had contracted by $68 \%$ of the initial diameter due to photo-induced dehydration of the polymer chain. Although the shrinking response was fast, the reswelling of the gel needed more than one hour, in the $0.05 \mathrm{mM} \mathrm{HCl}$ solution $[60,68-72]$.

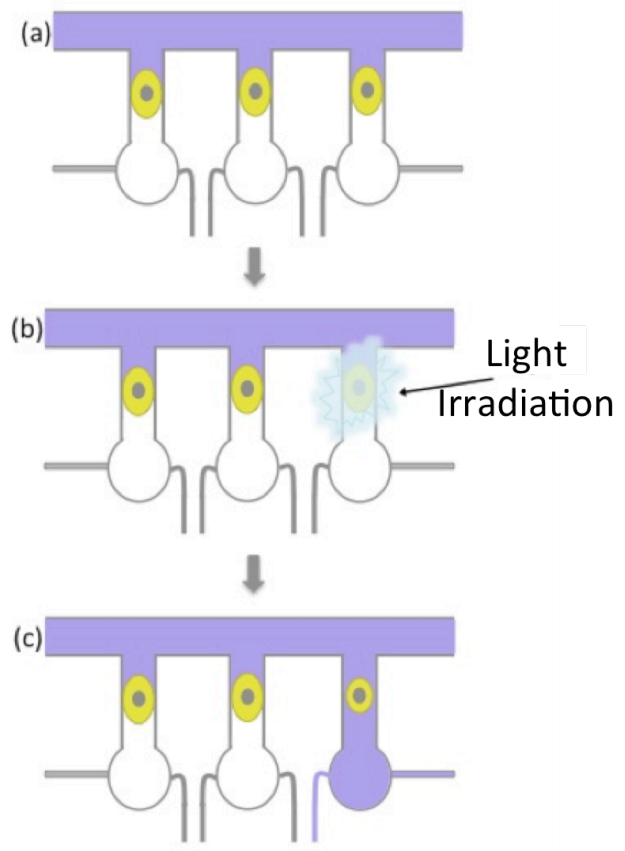

Figure 10. Illustration showing three parallel micro-channels each containing a photo-polymerised hydrogel valve; individual valve actuation can be achieved without interference with adjacent valves. (a) channels before valve photo-actuation, (b) during photo-actuation and (c) represents the channel after photo-actuation (Adapted from Sugiura et al. [69]). 
In order to improve the response time of p(NIPAAm-co-SP) hydrogels, ionic liquids (ILs) were introduced as plasticizers. Four different novel ionogels were used as photo-actuated valves in microfluidic manifolds. The spiropyran-functionalised ionogels were composed of pNIPAAm and phosphonium-based ionic liquids. The ionogels were photo-polymerized in situ, in micro-channels of a poly(methyl methacrylate) (PMMA) microfluidic device. Varying the ionogel composition meant that only one source of light was required for actuation (Figure 11). The response and visible contraction observed was very fast (seconds), while the re-expansion remained relatively slow (minutes). As each gel opened the channel at different times, such device allowed independent control of multiple microvalves. In this work it was found that the anion present in the ionic liquids directly affected the photoresponsiveness of the ionogel and thus the actuation behaviour; i.e. the actuation kinetics could be controlled to an extent by varying the anion of the phosphonium IL, with the $\left[\mathrm{NTf}_{2}\right]^{-}$exhibiting the fastest reponse. Although it was possible to reuse these valves, their long reswelling times and requirement for an acidic environment $(0.1 \mathrm{mM} \mathrm{HCl})$ made them a more appropriate candidate for single use devices [73].

(a)

5 inlets (with coloured dyes)

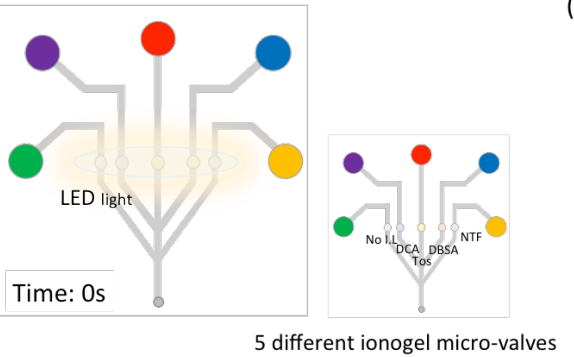

(b)

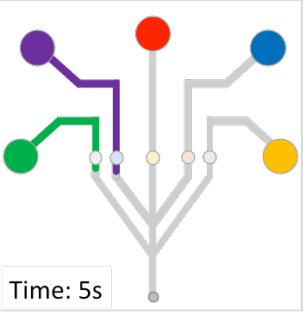

(c)

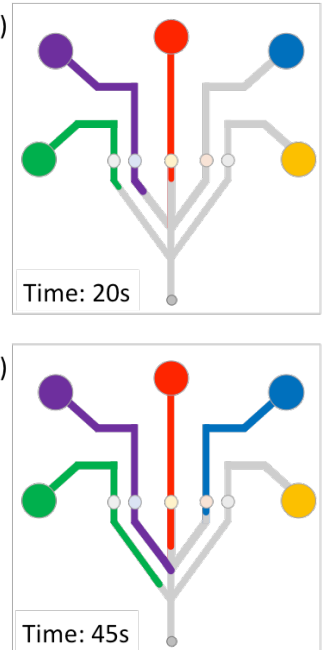

(e)

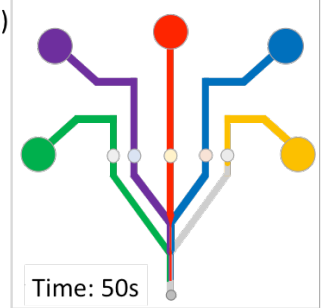

(f)

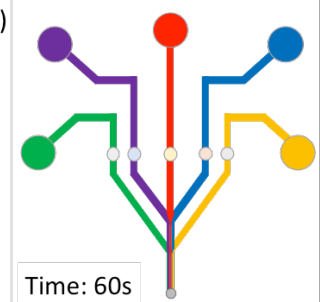

Figure 11. Illustration showing the performance of the ionogel micro-fluidic valves; a) micro-valves composed of the different ionogels are expanded and the valves are closed before light irradiation. $b$ )-d) depending on the anion incorporated inside the ionogel, each valves opens after different times of light irradiation. (Adapted from Benito-lopez et al [73]).

Ziółkowski et al.[66] demonstrated an acrylic acid moiety incorporated into the backbone of p(NIPAAm)-co-SP could provide an internal source of protons, enabling photo-responsive hydrogel behavior at neutral external $\mathrm{pH}$ (Figure 12). This study also investigated optimal acrylic acid:monomer ratio in the polymer, for improved light-induced volume changes. The study showed that these acrylic acid/spiropyrans modified gels (p(NIPAAm)-co-SP-co-AA ) could be used to make photo-controlled valves in a microfluidic system without the need for an acidic environment for reswelling [66]. In a related development, Dunne A. et al. [54] showed that the polymerisation solvent used can have a dramatic impact on the gel morphology, which also affects the light-induced actuation behavior of the resulting hydrogel.

Building on this research, Schiphorst J. et al. [67] further improved the actuation kinetics by varying the side chain on the spiropyran derivative. The improved formulation was then polymerised in-situ in a microfluidic channel to create a valve structure and exposed to blue light. Upon photoexposure for $1 \mathrm{~min}$ the hydrogel valves contracted, opening the micro-channel and allowing fluid to flow. When the light was removed, the hydrogel expanded, once again blocking the channel (Figure 13). This process was seen to be successfully repeatable for several actuation cycles. This type of photo-actuation offers a scalable, reversible and non-invasive form of control in microfluidic devices, which requires no direct contact with the material. 


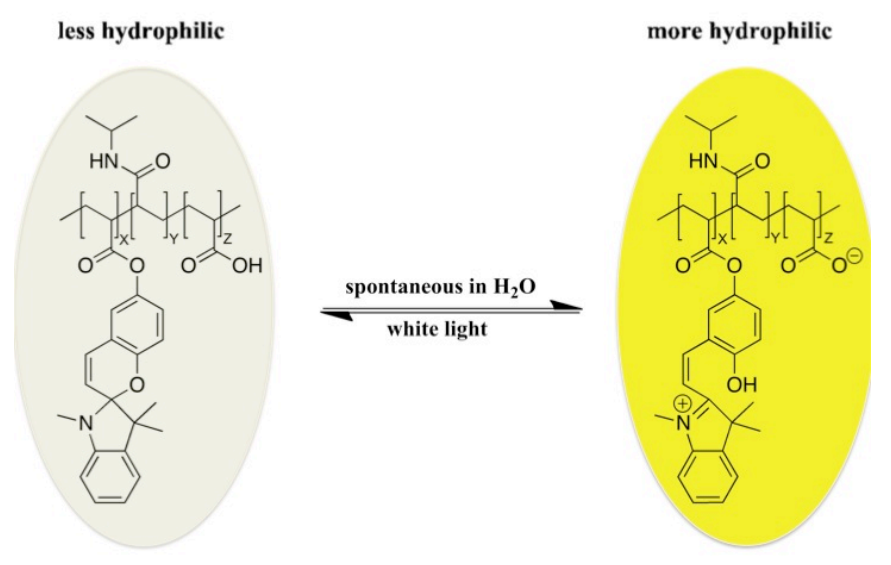

Figure 12. Representation of the proton exchange in the $p$ (NIPAAm)-co-SP-co-AA hydrogels, between the acrylic acid and the spiropyran moieties together with the effect of white light irradiation; (right) more hydrophilic chains containing the protonated, yellow coloured merocyanine $\left(M C-H^{+}\right)$; left: less hydrophilic chains obtained after white light irradiation due to the conversion of $\mathrm{MC}-\mathrm{H}^{+}$to the hydrophobic SP form;
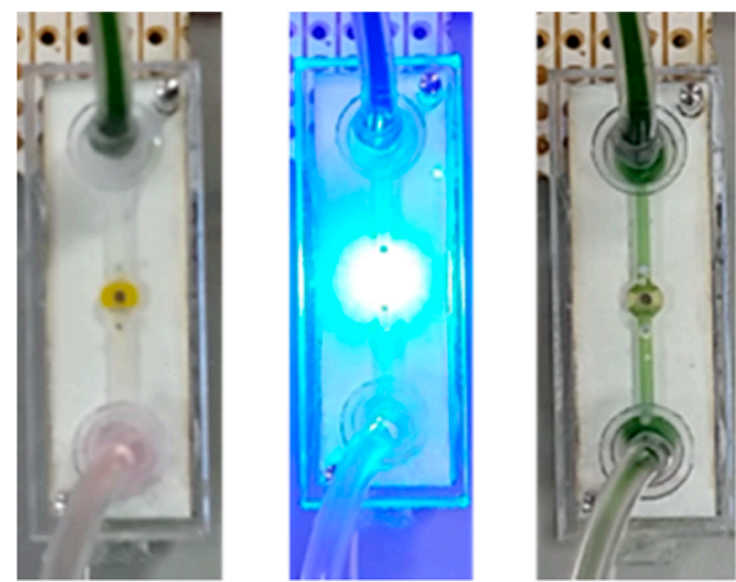

Figure 13. Photo-induced hydrogel valve actuation showing fluid flow modulation in a micro-channel; (left) p(NIPAAm)-co-SP-co-AA hydrogel is expanded and valve is closed; (middle) application of blue light; (right) hydrogel contracts upon light irradiation and valve opens allowing fluid to flow. (Reproduced from [67]).

\subsubsection{Magneto-induced actuation}

Another mode of stimulation used to control stimuli-responsive hydrogels in microfluidics is the application of a magnetic field. Hydrogels responsive to magnetic stimuli can be created by replacing the polymerisation solvent with a colloidal solution that contains nanometer to micrometer scale magnetic particles. Examples include magnetorheological fluids or Ferro fluids [74]. When a magnetorheological fluid is exposed to a magnetic field, its viscosity will increase, directly affecting the mobility of the polymer chains and the shape of the hydrogel. Magnetic stimulation has shown to work best when coupled with a thermally responsive polymer, such as p(NIPAAm) based hydrogels. After incorporation into a pNIPAAm hydrogel, the magnetic particles adhere directly to the polymeric network. When these hydrogels are exposed to an Alternating Magnetic field (AMF), the magnetic particles begin to heat up, directly affecting the pNIPAAm polymer. When the temperature increases to above the pNIPAAm LCST, the hydrogel transitions to the less hydrophilic state, resulting in water expulsion and hydrogel volume contraction [75]. (Figure 14) 


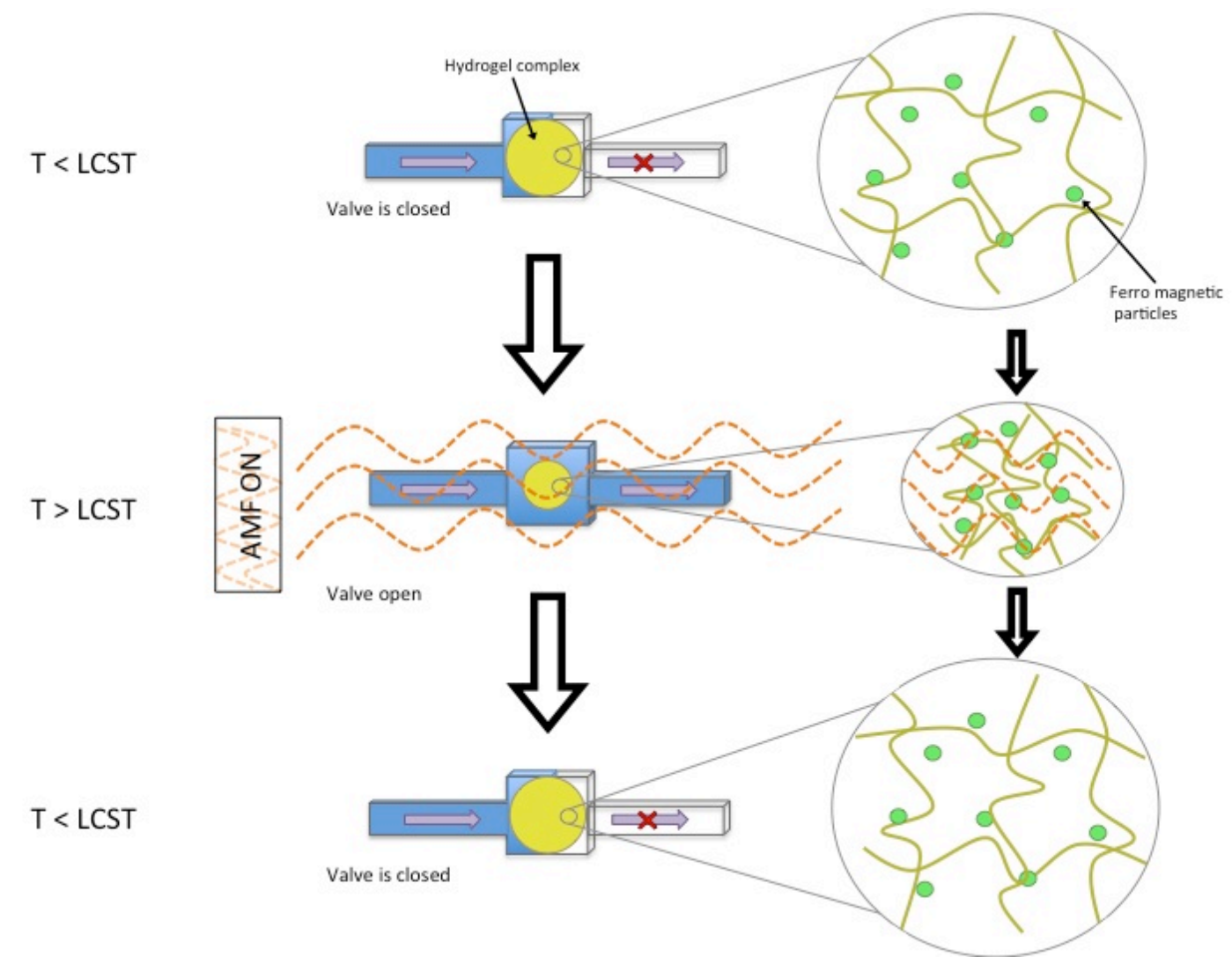

Figure 14. Schematic showing collapse of the hydrogel with the application of AMF, opening the valve. Adapted from N.S. Satarkar et al. [75].

Similarly, Ghosh et al. [76] have successfully demonstrated a magnetic-responsive ON/OFF system to control flow. The polymer material was composed of a p(NIPAAm) based hydrogel incorporating ferromagnetic nanoparticles $\left(\mathrm{Fe}_{3} \mathrm{O}_{4}\right)$. Using oscillating magnetic fields a hydrogel contraction of $\sim 80 \%$ was seen within $3 \mathrm{~s}$ of the magnetic field being applied. This study is consistent with the general rule that smaller scale hydrogel structures improve the kinetics of the actuation response. Most recently Hohlbein et al. have also presented a concept for on-demand self-healing elastomeric composites. Here, the magnetic nano-particles of different size, shape and composition were employed to act as nano-heaters, driven by a high-frequency AMF. This on-demand effect required very short times in order to 'self-heal' the elastomer, restoring its original shape [77].

Among the stimuli-responsive materials proposed for microfluidic applications described above, several advantages and disadvantages can be mentioned for each. For example, one great advantage of $\mathrm{pH}$-responsive materials is their internal response to the $\mathrm{pH}$ of the solution flowing inside the microchannel, without external intervention. Therefore, there is no need for the addition of external specialised equipment to control the actuation of such materials, as their response relies on localised $\mathrm{pH}$ changes. A disadvantage of $\mathrm{pH}$-responsive materials however, is the specific working $\mathrm{pH}$ range (typically 3-5 pH units), meaning that they are non-universal and have to be designed towards a specific application.

Thermo-responsive materials on the other hand, commonly exhibit fast response times and are generally independent of the working solution. However, they require external heating elements, which represents a significant hurdle for integration. Moreover, the increase in temperature can affect the surrounding solution and this can become problematic for biomedical applications, for example.

It could be then proposed that photo-responsive materials are possibly the most applicable for integration in microfluidic devices, primarily due to the nature of their autonomous (non-contact, external) actuation and the ease of integration of LED light sources in the microfluidic device, if 
desired. This permits selective actuation of individual photo-actuators with minimal energy consumption.

\section{Stimuli-controlled manipulation of synthetic discrete micrometer-sized "vehicles"}

Controlled movement of micro "vehicles" offers many intriguing and beneficial opportunities in the microfluidics field. For example, in droplet based microfluidic devices, the high surface area to volume ratio results in high heat and mass transfer rates. Also, most droplet microfluidic chips allow for control over individual droplets, thus allowing them to act as micro-vessels which can be mixed or merged with other droplets, moved to desired destinations or even individually analysed. These properties allow the droplets to have a diverse range of applications including micro-vessels for chemical reactions, dynamic sensing, cargo transport and potential drug delivery units [78, 79]. This section will focus on the most popular actuation methods employed thus far for stimuli controlledmovement of synthetic "vehicles" (e.g. droplets, beads, gels). Although the majority of the actuation methods described to date were not explored in microfluidics, the same concepts could be applied to work within a microfluidic system. These "vehicles" could also be used to mimic biological vesicles, which transport nutrients in and out of cells through highly regulated pathways, for drug transport and targeted delivery.

Actuation of liquids in the form of synthetic "vehicles" offers many advantages over conventional laminar flow microfluidic systems, which rely upon the use of pumps and valves to control and direct flow, while stimuli-controlled droplet microfluidic systems allow for external manipulation of individual or multiple droplets simultaneously. These "vehicles" can act as microreactors, cargo-carriers, and can be used to transport or store volumes of reagents down to the picoliter range $[80,81]$.

Actuation of droplets can be categorised based on two main mechanisms. The first mechanism (and the most widely used) involves control over the wettability of a substrate, such as polymeric plastics (e.g. polydimethylsiloxane (PDMS), poly(methyl methacrylate) (PMMA)), glass and silicon wafers, which allows for unidirectional movement of droplets over the substrate. One of the most popular droplet actuation methods based on this principle is known as digital microfluidics (DMF). In DMF small discrete droplets are transported across an array of individually addressable electrodes, by controlling the displacement of the droplet through localised control of surface polarisation and wettability. Additionally, many research groups have explored the use of different alternative stimuli such as light [82-87], temperature [88-93] and magnetic stimuli [94-99] for wettability control.

The second mechanism involves manipulation of the surface tension of a liquid. This allows controllable movement of droplets or other "vehicles" at the liquid/air interface. To control the surface tension of a liquid, surfactants are used. Through the asymmetrical release or alteration of the surface activity of stimuli-responsive surfactants, surface tension gradients can be created around the droplet. This in turn results in Marangoni flows, through which liquid spontaneously moves from areas of low surface tension towards areas of high surface tension. Thus, through contactless control over the surface tension of an aqueous solution, unidirectional movement of a droplet resting at the liquid/air interface can be achieved. This type of surfactant driven movement can be broken down into two main actuation mechanisms, either by addition of the surfactant to the aqueous medium or inclusion of the surfactant in the 'vehicle'. In the first approach, with stimuli-responsive surfactants dispersed in the aqueous solution, application of an appropriate stimulus to the surfactant alters its surface activity, which results in a change in the local surface tension. This in turn causes a flow of liquid towards areas of higher surface tension; any droplet or "vehicle" resting on the surface of the liquid will be consequently transported in the direction of the flow. In the second approach, the surfactant is contained within the "vehicle" itself and its asymmetric release is externally controlled, resulting in a surface tension gradient around the droplet, which triggers unidirectional droplet movement. 


\subsection{Actuation through localised changes in wettability}

This concept involves moving droplets on a substrate to targeted areas, through changes in the wettability of the substrate. Numerous reviews and articles have been published in this area in the past decade which provide comprehensive overviews of the topic [79, 100, 101]. This section will focus on the "vehicle" movement mechanism and the different means for achieving "vehicle" actuation. Several applications will be mentioned and discussed.

\subsubsection{Digital Microfluidics}

In DMF, droplets are actuated on an array of electrodes which are individually addressable. Using this array of electrodes, a wettability gradient can be generated on the substrate by applying different voltages between adjacent electrodes. The droplet can be then moved along this electro-generated wettability gradient to a desired destination by continually altering the potential difference between neighbouring electrodes. The droplets are produced from reservoirs within the chip and they can be split, mixed with other droplets or simply used to transport cargo inside the chip [100]. There are two types of actuation mechanisms required for this mechanism; the first involves the initial generation of the droplets from a reservoir and the second is needed for movement of the droplets through the system. The two main methods of electro-chemical control over droplet formation and movement in DMF systems are dielectrophoresis (DEP) and electrowetting on dielectric (EWOD). Alternative actuation for DMF utilizes thermocapillary transport, surface acoustic wave transport (SAWS) and optical forces and magnetic forces [100, 101].

Applications of DMF systems include bio-assays [102], DNA applications, such as polymerase chain reactions (PCR) [103, 104], separation and analysis of aminoacids, peptides and other biological molecules [105], cell based assays [106], proteomics [107], and adaptive cooling of integrated circuits systems [108].

\section{- Electrowetting on dielectric}

The phenomenon termed electrowetting on dielectric (EWOD) is one of the main forms of actuation associated with DMF. In EWOD the interfacial tension between an aqueous droplet and electrode is electrically altered in such a manner as to actuate the droplets across a series of electrodes. When a voltage is applied, the interfacial energy between the droplet and electrode surface is lowered, changing the wettability of the substrate [79, 100]. Voltage range varies greatly depending on the application, but typically the values lie between $0-200 \mathrm{~V}$ [109-111]. For example a micro-sized droplet was split into two daughter droplets by applying a potential difference of $25 \mathrm{~V}$ between adjacent electrodes [109]. Interfacial energy directly affects the contact angle; by lowering the contact angle between the droplet and the surface, an interfacial energy gradient is created which actuates the droplet. By applying voltages to individual electrodes an interfacial energy gradient will be formed which the droplet follows [112]. In EWOD there are generally two possible set-ups, namely a single plate or a two-plate system. In a single plate (or open plate) system the droplet sits on a substrate, which contains both actuation and ground electrodes. The two-plate configuration is a closed system in which the droplet is held between two substrates. The top substrate contains a continuous ground electrode while the bottom layer houses the actuation electrodes. Figure 15 is a representation of a typical a) closed, b) open and c) operation of both open plate (single) and closed plate (double) DMF systems. In both cases the layer containing the actuation electrodes must be insulated and covered by a layer of hydrophobic material. The hydrophobic material ensures that the aqueous solutions do not wet the surface and thus form droplets; it also ensures that the droplets have a high contact angle with the substrate. Using the actuation electrodes this contact angle can be altered which can result in unidirectional movement of the droplet [112]. 
(a)

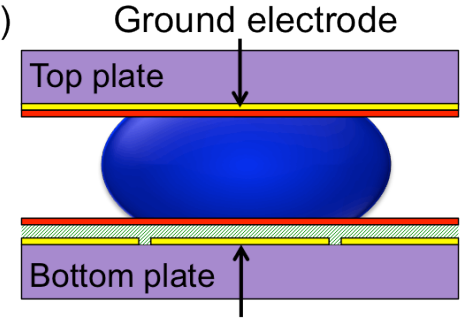

Actuation electrode

(b)

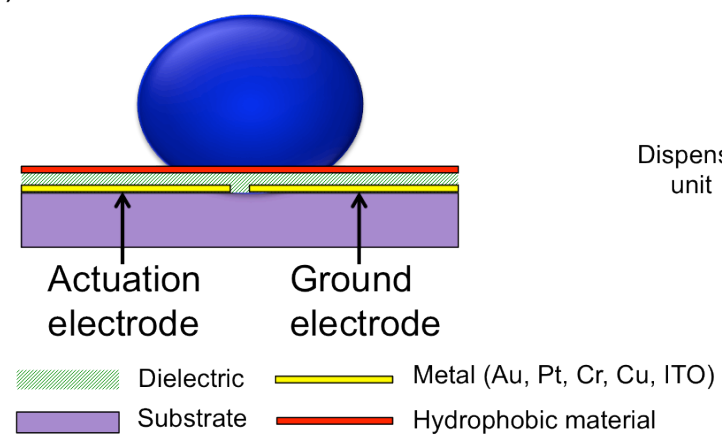

(c)

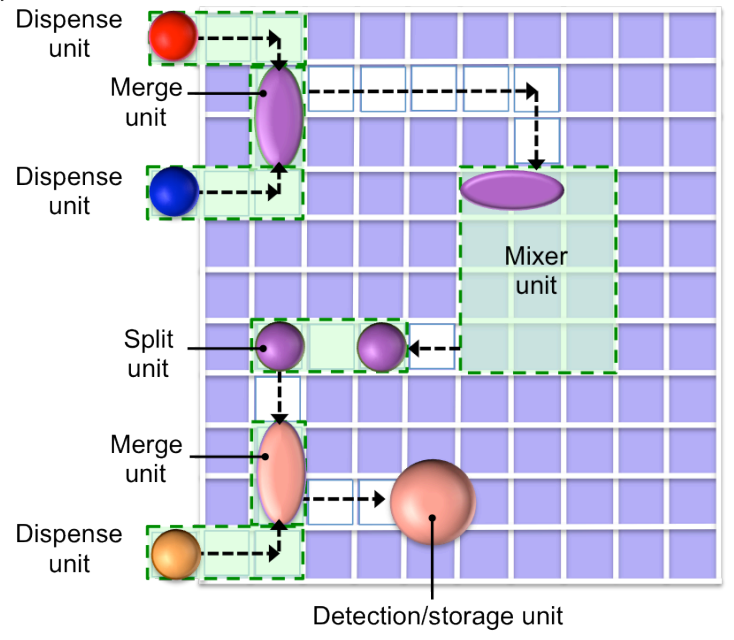

Figure 15. (a) Closed/two plate DMF system. (b) Open/one plate DMF system. (c) basic DMF operations. Adapted from [112] with permission from The Royal Society of Chemistry.

\section{- Dielectrophoresis}

Dielectrophoresis (DEP) is one of the most popular techniques for the manipulation of droplets in DMF. DEP works by pulling (positive DEP) or pushing (negative DEP) a dielectric particle away from the actuation electrode. In a DEP driven system the particle does not necessarily need to a have a charge, as all particles will exhibit electrostatic properties when introduced to an electric field. Typical frequencies for DEP driven systems are between $100 \mathrm{~Hz}-10 \mathrm{MHz}[113,114]$. In DEP the particles are subjected to a non-uniform electric filed, creating a dipole moment on the particle, that causes the droplet to be electrostatically pulled towards the actuated electrode. The size and uniformity of the droplets being formed in this manner depends on the magnitude and frequency of the applied voltage [115].

\subsubsection{Alternative methods for substrate droplet actuation}

Many research groups have explored alternative methods for achieving droplet actuation on a solid substrate. Although DMF is a great tool for the manipulation of discrete volumes of liquids in microfluidics, the chip designs are complicated and energy is required to move the droplets. The following sections will focus on alternative methods which use various types of stimuli for moving droplets on a solid substrate.

\section{- Photo-induced actuation}

Optoelectrowetting (OEW) has a similar mechanism to EWOD, however in OEW a photoconductive layer (generally amorphous silicon) is inserted between the dielectric layer and the electrodes layer. Droplet actuation is achieved when a voltage is applied across the insulating layer of the device. In darkness, the resistance of the photoconductive layer is high, resulting in the insulating layer receiving no voltage, meaning that the contact angle between the droplet and the substrate remains unchanged and therefore no actuation is achieved. Upon illumination, the conductivity of the photoconductive layer increases. This results in the insulating layer receiving the applied voltage. In this fashion the contact angle of the droplet can be controlled with light and actuation can be achieved [84]. When an 
optical beam is used to irradiate on one end of the droplet, the contact angle is reduced in such a way that the pressure difference between both ends causes the droplet to follow the light beam. This was firstly demonstrated by Chiou et al. [84] in 2003 when a droplet was successfully actuated using a 525 $\mathrm{nm}$ laser with intensity of $65 \mathrm{~mW} / \mathrm{cm}^{2}$. The difference between an OEW device and a conventional EWOD device can be seen in Figure 16.

(A) Conventional EWOD Device

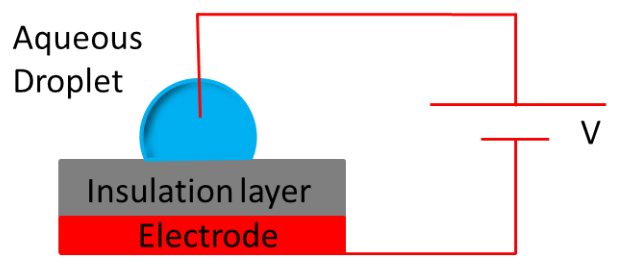

(B) Optoelectrowetting (OEW) Device

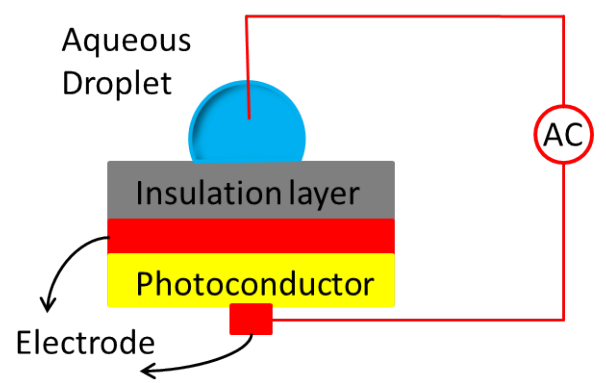

Figure 16. Schematic of (a) conventional EWOD and (b) an OEW device. The major difference between the two systems is the photoconductive layer inserted between the electrode and insulating layer in an OEW device. Adapted from [84].

Since Chiou and co-workers [84] successfully demonstrated photo-induced droplet actuation by using a photoconductive layer, many research groups have explored various means of photo-actuating droplets. Wereley et al. [85] developed a novel open-Optoelectrowetting (o-OEW) system which used a $670 \mathrm{~nm}$ laser with an intensity of $15 \mathrm{~mW} / \mathrm{cm}^{2}$. Wereley's OEW system actuates droplets through photo-induced electrowetting of a substrate using a photoconductive layer, much like Chiou's device. However, in the chip designed by Wereley, all the electrodes needed for droplet actuation are contained within a single substrate. This open fluidic chip allows easier integration with additional components or chip extension.

Photo-induced actuation of droplets can also be achieved by creating photo-responsive surfaces. In this manner the hydrophobicity of the surface can be controlled through photo-stimulation. Various photo-responsive surfaces have been created by functionalising glass or polymeric surfaces with photo-sensitive units. Examples of widely used photo-sensitive molecules include spiropyrans and azobenzenes [87]. Under photo-irradiation these photo-responsive materials are reversibly switched between two isomers of different hydrophobic character and surface activities. When immobilised on surfaces, switching between the different isomers directly affects the wettability of the substrate allowing for photo-controlled droplet actuation. Rosario et al. [116] demonstrated actuation of water droplets across a rough and flat silicone surface coated with hydrophobic monolayers which contained photochromic spiropyrans. Under visible light, the spiropyran molecule was in its closed spiropyran (SP) hydrophobic state however upon UV light $(366 \mathrm{~nm})$ irradiation, the SP isomer was switched to the more hydrophilic merocyanine (MC) form, reducing the contact angle between the water droplets and the surface. Therefore unidirectional movement of the droplets was achieved through the use of UV/visible light generated wettability gradients.

Azobenzenes are another popular photochromic group, due to their reversible photoisomerization between cis and trans isomers. Azobenzenes were first used to control the wettability of a substrate by Siewierski et al. [117] in 1996. The paper describes two methods for 
preparing silicon surfaces coated with azobenzene monolayers. Irradiation of the monolayers with light of $354 \mathrm{~nm}$ resulted in the isomerisation of the azobenzene within the monolayer to the cis conformation. This resulted in a decrease in the contact angle between water and the substrate. The contact angle difference in the case of all the monolayers were in the range of $2^{\circ}-9^{\circ}$ and the results where repeatable. The greatest photo-induced contact angle change was observed for a film prepared by acylation and had pentyl terminal groups. Under ambient laboratory conditions (in trans configuration) the azobenzene monolayer film had a contact angle with water of $85^{\circ}$ while after UV irradiation (cis configuration) the contact angle was lowered to $76^{\circ}$. Since 1996 there has been much interest in azobenzenes and many research groups have used them for photo-actuation. Oh et al. [118] achieved photo-controlled directional movement of an oil droplet on an azobenzene-terminated monolayer modified silica substrate. This was realised by photo-isomerizing the azobenzene unit from the cis (contact angle of $11^{\circ}$ ) to trans (contact angle of $24^{\circ}$ ) conformation through asymmetrical blue light $(436 \mathrm{~nm})$ irradiation. Movement of the light source allowed for continuous droplet movement. The direction and speed of the droplet was dependent on the steepness and intensity of the light gradient.

\section{- Thermo-induced actuation}

Thermo-induced actuation provides an alternative method for controlling droplet movement on a substrate without chemical surface functionalization. This type of movement is known as thermocapillary transport of droplets. Droplet actuation is induced from the temperature variation which arises at the liquid/gas interface of the droplet, when placed on a solid substrate which has a temperature gradient. The temperature variation between the liquid/gas interfaces creates a surface tension $(\delta)$ gradient around the droplet from the warm (low $\delta$ ) to the cool side (high $\delta$ ). This asymmetrical gradient creates a flow within the droplet which applies a hydrodynamic force on the substrate. Since the solid surface is held in a fixed position it produces an equal and opposite force which actuates the droplet driving it to the cooler region [119]. Darhuber et al. [93] demonstrated that thermocapillary actuation can be used for droplet formation as well as moving droplets.

\section{- Magnetic-induced actuation}

Magnetic-induced actuation of droplets is achieved through the use of magnetic materials in various forms. A popular approach is to use liquid marbles, which are aqueous droplets that are rendered completely non-wetting by coating with magnetic hydrophobic powders. Since the powders are hydrophobic they are immiscible with the droplet and therefore adhere to the exterior of the droplet. This results in a liquid droplet which does not stick to smooth substrates and can be manipulated using magnetic fields. Many research groups used iron or iron oxides micro-particles (due to their ferromagnetic properties) as the hydrophobic powders in order to create the liquid marble [94]. Zhao et al. [96] demonstrated magnetic manipulation of a liquid marble on a glass substrate by coating their droplet with highly hydrophobic Iron(III) oxide $\left(\mathrm{Fe}_{3} \mathrm{O}_{4}\right)$ and placing a magnetic bar at one end of a glass substrate. Droplet actuation was achieved by slowly moving the magnet towards the droplet until this began to move. The droplet could then be made to follow the magnetic bar (by slowly moving the bar away from the droplet) across the glass substrate. Zhao and co-workers also demonstrated that the marble particles could be pulled from the liquid by placing a strong magnet directly under the droplet on the glass slide. To prove this, the group coated a water droplet containing a blue dye with $\mathrm{Fe}_{3} \mathrm{O}_{4}$. Once the droplet was coated, the dye was not visible. When the magnet was placed below, the droplet's coating was attracted to the bottom of the droplet, hence showing the dye. When the magnet was removed, the $\mathrm{Fe}_{3} \mathrm{O}_{4}$ nanoparticles quickly moved back to the exposed liquid to reform the liquid marble. 


\section{- Surface acoustic waves}

Actuation of droplets can also be realised using surface acoustic waves (SAWs). In this case, actuation is achieved by producing acoustic radiation pressure on the surface of the substrate. This leads to an internal flow within the droplet and to eventual droplet actuation [120]. Wixforth et al. [120] demonstrated actuation of a droplet across a piezoelectric chip through the use of SAW pumps. The chip was designed to have virtual beakers and channels which confined the liquid to the surface. Since the acoustic waves were electronically addressable they were able to act as programmable nanopumps. Another example of using SAWs to move droplets across a substrate was demonstrated by Guttenberg et al. [121]. Guttenberg developed a planar chip for polymerase chain reactions (PCR) and hybridization which utilizes SAWs for the movement of micro-droplets. The microfluidic device used had a planar piezoelectric Lithium niobate $\left(\mathrm{LiNbO}_{3}\right)$ substrate. The chip was chemically modified to produce high contact angles between the substrate and the droplet thus ensuring that the droplet movement was controlled through the SAW pump. The chip also contained thin film resistance heaters which provided the energy needed for the PCR reactions.

\subsection{Actuation through localised changes in surface tension}

This type of actuation in the microfluidic sector is a relatively new concept compared to actuation through changes in wettability and involves generation and transport of droplets across liquid environments.

To achieve actuation through localised changes of surface tension at the liquid/liquid, liquid/gas interface, surfactants have been used as these have the unique property of modifying the surface tension of a liquid [122]. Although many stimuli-responsive surfactants have been synthesised, only a small number have been used for actuation of droplets or other micro-vehicles [123].

In the bulk of a liquid, the attractive forces between molecules are shared by all neighbouring molecules and no net force is felt as they cancel each other out. However molecules at the interface (liquid/gas, liquid/liquid) have no attractive forces been exerted from above, therefore they exhibit stronger forces between the nearest neighbouring molecules at the surface. This causes the molecules at the interface to contract forming a contractile layer (Figure 17). This contractile layer at the surface is known as the surface tension $(\gamma)$.

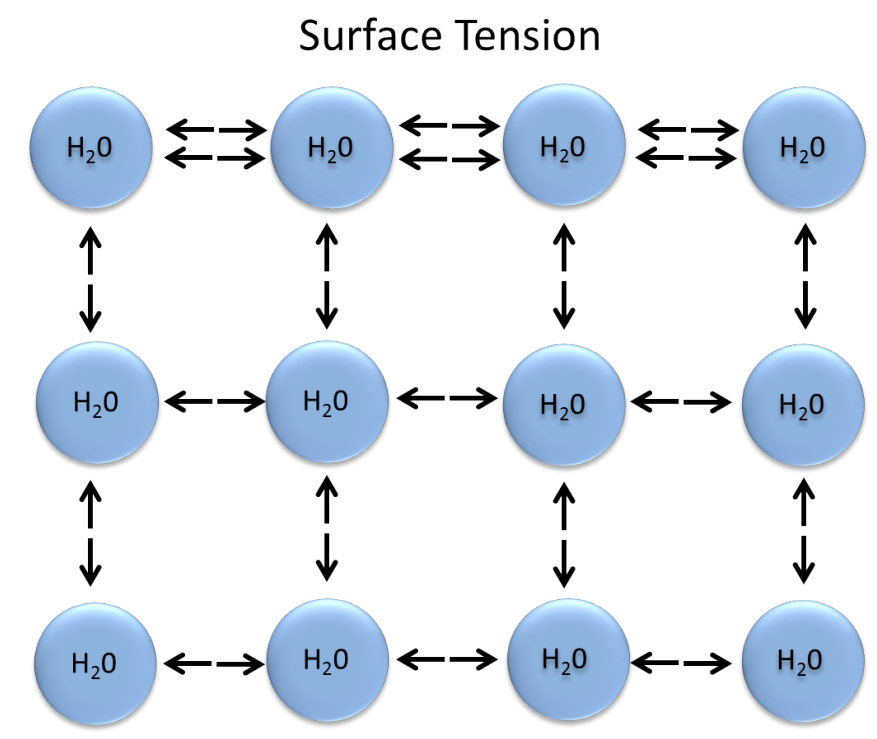

Figure 17. Representation of the net forces in water.

Certain molecules have special properties whereby they can imbed themselves at the interface and interrupt this elastic layer reducing the local surface tension. These molecules are known as surfactants. The term surfactant comes from surface active agents. Surfactants are molecules which are 
generally long chained amphiphiles which have a polar "head" and a non-polar tail. In an aqueous solution (below a certain concentration known as the critical micelle concentration (CMC)) the surfactant's polar head will interact with the polar molecules at the surface. This interaction will interrupt neighbouring attractive forces and lower the surface tension of the solution.

Fluid flows from areas of low to high surface tension $(\gamma)$. This is known as the Marangoni effect. The Marangoni effect describes the mass transfer along an interface between two fluids due to a surface tension gradient. This causes convective flows in a bulk solution as well as inside of a "vehicle". The following sections describe systems which take advantage of this effect to achieve activation of droplets along the liquid/gas interface.

\subsubsection{Surfactant saturated systems}

A surfactant saturated system is one in which surfactants are dissolved in the solution up to their particular CMC. This ensures that the surfactant molecules are concentrated at the interface instead of forming micelles. Marangoni flow can be induced if the surface tension of a targeted area is controlled. In order to induce movement through changes of the surface tension, the surfactant's surface activity has to be altered. To control the surface activity, stimuli-responsive surfactants are used [124]. In this fashion, if the correct stimulus is applied to a targeted area of the system, Marangoni flows can be induced on demand. Photo-responsive surfactants have been used to externally control the surface tension of aqueous solutions. Direct irradiation of a targeted area is relatively simple and wavelengths can be tuned for particular molecules. Photo-responsive surfactants possessing two isomers with different surface activities have been shown to work in this fashion. Unidirectional movement of a micro-vehicle resting on an aqueous solution that contains photo-sensitive surfactant is achieved by irradiation of a specific area around the vehicle. This breaks the surface tension symmetry around the droplet and creates the Marangoni-like flows which drive the droplet either away or towards the light source. An example of a photo-switchable azobenzene surfactant (AzoTAB) is shown in Figure 18.

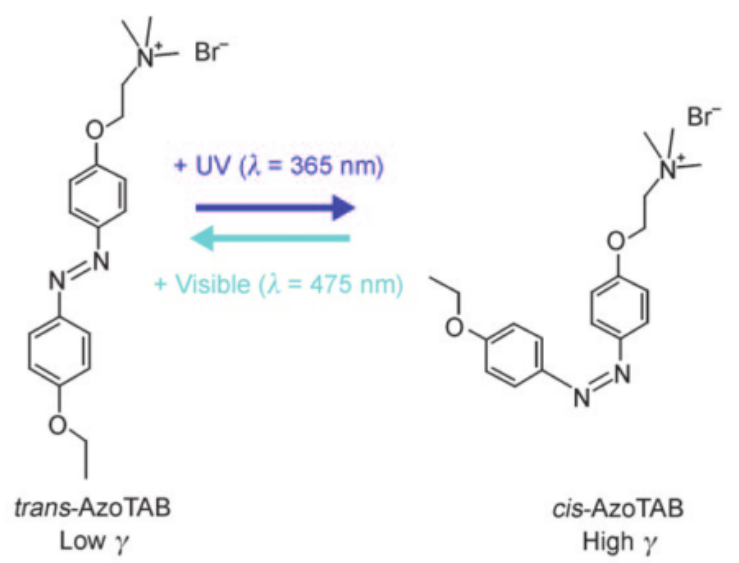

Figure 18. Photo-induced confirmation change of AzoTAB between its two isomers: from trans (high $\gamma$ ) and to cis (low $\gamma)$. Adapted from [125] with permission from The Royal Society of Chemistry.

Diguet et al. [126] demonstrated photo-triggered movement using an oleic acid droplet resting on an aqueous solution containing the AzoTAB surfactant (Figure 18). The droplet of oleic acid can be moved by irradiating half of the droplet with either $365 \mathrm{~nm}$ or $475 \mathrm{~nm}$ light. When the AzoTAB surfactant is exposed to $365 \mathrm{~nm}$ of light, it is made to isomerise to its cis configuration. In this configuration the AzoTAB has a higher surface tension $\left(8 \mathrm{mN} \mathrm{m}^{-1}\right)$ than in the trans configuration (7 $\mathrm{mN} \mathrm{m}^{-1}$ ). Therefore, when half of the droplet was illuminated with $365 \mathrm{~nm}$ light it was made to follow the light source as the surface activity of the surfactant was higher compared with the non-irradiated section. When the droplet was irradiated with light of $475 \mathrm{~nm}$, the AzoTAB isomerised to its trans 
configuration and thus lowered the surface tension of the solution, which resulted in the droplet being repelled by the light.

In order to achieve continuous droplet movement, the droplet was followed with the light source or trapped using a trap consisting of both light wavelengths (365 nm and $475 \mathrm{~nm}$ ). Multiple droplets were also manipulated in a petri dish in this fashion.

Photo-actuation can also be used to control the flow within a microfluidic system. Recently it was demonstrated that the AzoTAB surfactant could be used to change the flow in a microfluidic system from laminar to segmented flow (droplets) [125]. Initially the system had a two-phase flow, one flow was aqueous (which contained the AzoTAB surfactant) and the second was an oil phase. When the aqueous phase was illuminated with light of $365 \mathrm{~nm}$, the AzoTAB isomerised to its cis configuration and the interfacial energy of the aqueous solution on the substrate was increased which fragmented the solution into monodisperse droplets (Figure 19). When the light source was removed, the flow returned to laminar. This was a reversible process and the flow was successfully switched multiple times in the same experiment [125].

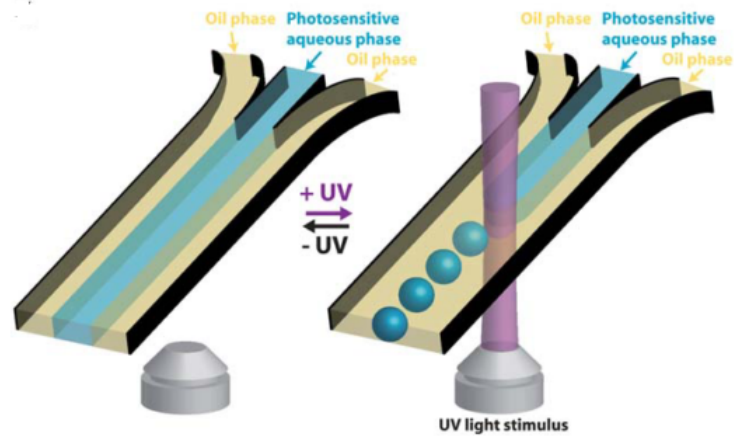

Figure 19. Cartoon illustrating the photoreversible fragmentation of a two phase (aqueous/oil) liquid flow. Reproduced from [125] with permission from The Royal Society of Chemistry.

A recent study by Toyota et al. [127] demonstrated an autonomous droplet which spontaneously moved once placed in an aqueous solution in which "fuel" surfactant had been dispersed. The surfactant used was (N-(4-[3-[trimethylammonio]ethoxy]benzylidene)-4-octylaniline bromide) while the droplets contained a catalyst which hydrolysed the surfactant. The self-propelled movement of these droplets can be described in three stages. The first stage involves the hydrolysis of the "fuel" surfactant at the surface of the oil droplets (which contained the catalyst). During the hydrolysis of the "fuel" surfactant there was a fluctuation of the rate of hydrolysis and this caused a symmetry-breakage of all the potential reactive sites on the surface of the droplet. This resulted in the hydrolysed product being accumulated at the most reactive sites. The second stage involved an imbalance of interfacial tension being created between the side of the droplet which accumulated the product and the side of the droplet which did not accumulate any product. Because of this imbalance of interfacial tension, lateral movement of the hydrolysed product was created on the surface of the droplet. The hydrolysed surfactant did not dissolve into the initial droplet since it was lipophilic and instead it aggregated to form small waste droplets. As a consequence, the waste droplets grew in size until they became too large and were subsequently released from the droplet (Figure 20). The interfacial energy of the leading edge of the droplet was lower than the waste covered trailing edge, creating interfacial dynamic fluctuation. This fluctuation combined with the accumulation and release of product resulted in the self-propelled motion of the droplets. In the final stage the leading edge of the droplet would pick additional fuel surfactant which was then converted into the lipophilic product as the droplet moved. This continuous collection and hydrolysis of the fuel surfactant further fed the interfacial imbalance and created internal convection inside the droplet, thus sustaining droplet movement. As long as there was "fuel" surfactant within the aqueous solution and the catalyst inside of the droplet was active, the droplet would continue to move. Although in this paper the system is not in a micro-channel nor is it controlled by external stimuli, this report opens up new avenues for stimuli- 
responsive actuation in a micro-channel. If a chemical reaction can be triggered by external stimulation then precise unidirectional movement can be achieved.
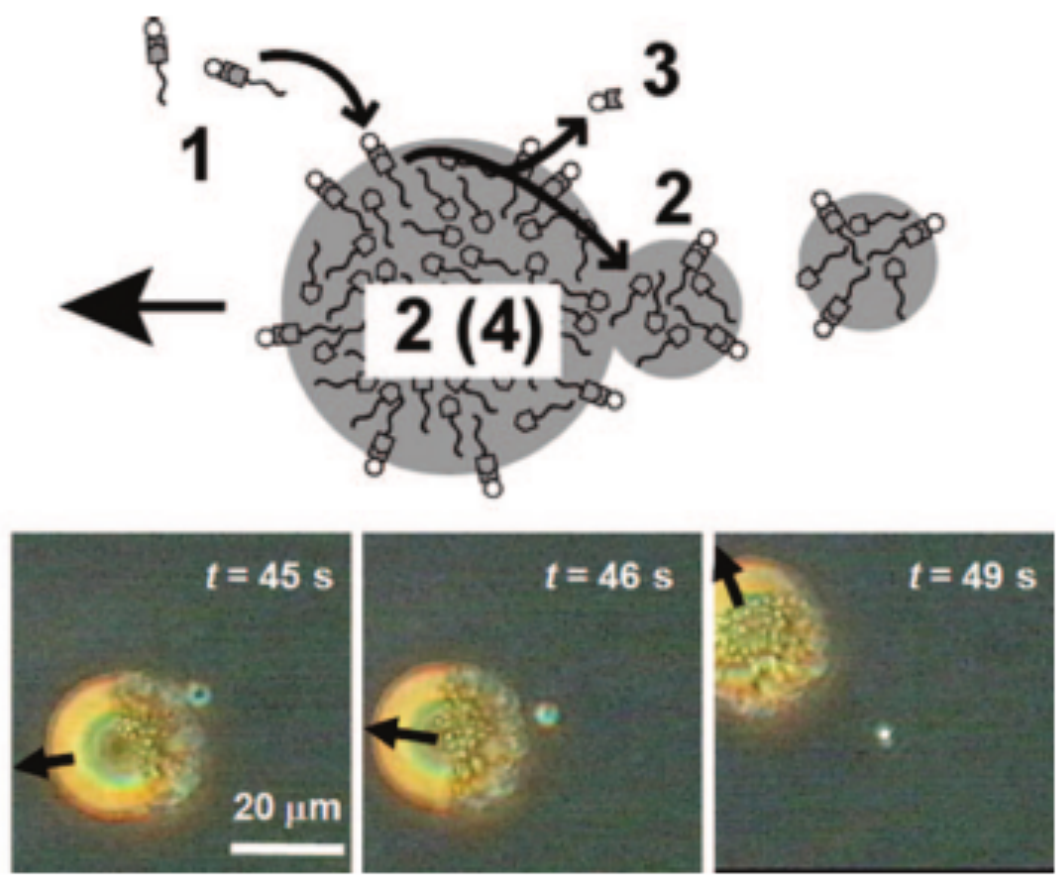

Figure 20. (a) Illustration of reaction happening on the exterior of the droplet and (b) fluorescent images of the droplet in motion. Reprinted with permission from [127]. Copyright (2009) AmericanChemical Society.

Jitka Čejkova et al [128] recently reported a simple decanol droplet system which is capable of mimicking a variety of biological process including reversible chemotactic movement, the ability to detect and move towards the strongest source of chemoattractant, stimuli responsive chemotactic movement and delivery of chemical cargo. In this study the decanol droplet is able to sense and move across aqueous solutions of sodium deaconate in the direction of $\mathrm{NaCl}$ gradients (Figure 21).

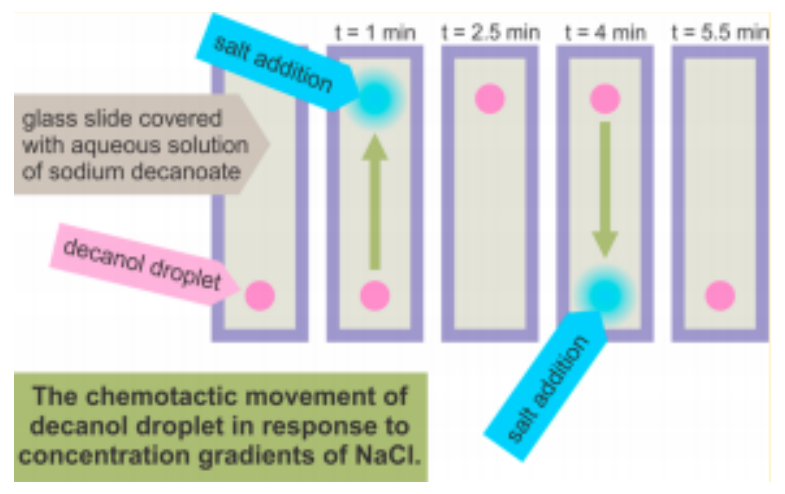

Figure 21. Illustration representing the reversible chemotactic movement of decanol droplets in $\mathrm{NaCl}$ gradients. Reprinted with permission from [128] Copyright (C) 2014, American Chemical Society.

The chemotactic movement is a result of Marangoni type convection forces developed as a result of interfacial tensions gradients caused by the addition of $\mathrm{NaCl}$. As the $\mathrm{NaCl}$ diffuses into the aqueous solution, it dilutes the amount of sodium deaconate molecules thus raising the surface tension of the solution resulting in a surface tension gradient leading to the source of addition. When no $\mathrm{NaCl}$ source is present in the system the decanol droplets move in random motion, this is due to the dissolution of the droplet itself into the aqueous solution (decanol is a weak surfactant). To achieve reversal chemotactic movement of the decanol droplet, the group developed a system of time delayed 
additions of salt (Figure 21). This ensures that a stronger $\mathrm{NaCl}$ gradient was created with each new addition, which led to the droplet moving to the new site.

To test whether the droplet would travel towards a stronger source of chemoattractant, the group dissolved varying concentrations of $\mathrm{NaCl}$ in nitrobenzene. This had the added advantage that the gradient could be maintained for longer due to the immiscibility of the nitrobenzene with the aqueous solution. To test whether the droplet would "choose" a chemoattractant source based on strength, a simple maze channel was designed, where in one end of the channel a nitrobenzene droplet containing $10 \mu \mathrm{mol}$ of $\mathrm{NaCl}$ was placed and at the other end of the channel a droplet which contained $50 \mu \mathrm{mol}$ was placed (Figure 22). In every run the droplet always chemotactically moved towards and merged with the most concentrated $\mathrm{NaCl}$ source.

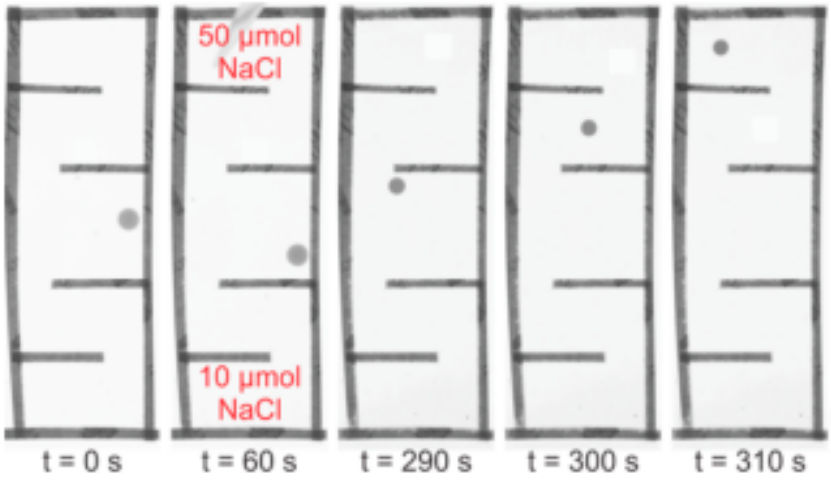

Figure 22. Snapshots showing that the decanol droplets were attracted by the stronger NaCl source. Reprinted with permission from [128] Copyright (C) 2014, American Chemical Society.

To demonstrate stimuli-responsive chemotactic movement the group encapsulated small $\mathrm{NaCl}$ salt crystals in paraffin particles which had a melting point of $42^{\circ} \mathrm{C}$. When the paraffin particles were placed into a solution of sodium deaconate below this temperature, no chemotactic movement was observed. However when the area around the wax particles was locally heated to above this temperature, the wax melted and slowly released the salt crystals which dissolved into the aqueous system and resulted in the chemotactic movement of the decanol droplets.

Finally the authors tested whether it was possible to carry and delivery chemical payloads. To do this, the decanol droplets where saturated with solid iodine which produced dark orange droplets. $\beta$ carotene was added to the nitrobenzene droplets which produced a red colour. The nitrobenzene droplet was placed at one end of a simple maze channel and the decanol droplet was placed at the start; once the decanol droplet chemotactically found and merged with the nitrobenzene droplet a green colour change was observed which indicated the iodination reaction of the $\beta$-carotene.

\subsection{2. "Vehicle" contained surfactants}

Movement of "vehicles" which contain the "fuel" surfactant is achieved by only releasing the surfactant upon external stimulation, as opposed to having the surfactant dispersed in the aqueous solution. This ensures that the surface tension is only modified around the droplet upon surfactant release, resulting in self-sufficient droplets. Vehicles that use this type of propulsion mechanism will continue to move as long as both the stimulus and the surfactant are present in the system.

Grzybowski et al. demonstrated this effect by developing smart droplets which were capable of solving complex mazes through the triggered release of a $\mathrm{pH}$ sensitive surfactant [129]. The surfactant used was 2-hexyldecaonic acid (HDA). When the HDA was subjected to a basic solution it was deprotonated to a more surface active form, $\mathrm{DA}^{-}$. The surfactant was contained within an oil or organic solvent such as dichloromethane. The droplet was able to solve the maze by following a $\mathrm{pH}$ gradient. This was achieved by filling the maze with a basic solution $(\mathrm{pH} \mathrm{12)}$ and placing an acidic gel at the end of the maze, creating a $\mathrm{pH}$ gradient. When the droplet was placed at the start of the maze (high $\mathrm{pH})$ the surfactant diffused out of the droplet into the solution. Since the $\mathrm{pH}$ was higher behind the droplet than in front of it, more $\mathrm{DA}^{-}$was generated behind the droplet (Figure 23). This created a 
surface tension gradient around the droplet, which in turn generated Marangoni like flows in the solution and also within the droplet. These flows pushed the droplet towards the area of highest surface tension, which was the exit of the maze (location of the acidic gel). As the droplet approached the exit of the maze it began to slow down as the concentration of $\mathrm{DA}^{-}$decreased due to the lower $\mathrm{pH}$ of the solution. However, as there was always a higher concentration of the $\mathrm{DA}^{-}$behind the droplet, the surface tension gradient was maintained, driving the droplet towards the exit of the maze.

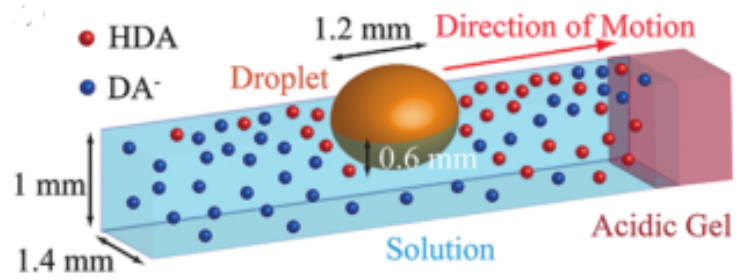

Figure 23. Distribution of HDA and DA around a droplet in a $\mathrm{pH}$ gradient. Reprinted with permission from ([129]). Copyright (2010) American Chemical Society.

Using the same droplet composition, self-dividing droplets were also demonstrated [130]. These self-dividing droplets were highly $\mathrm{pH}$ sensitive and only divided if placed on a highly basic solution. Actuation in this instance was very similar to the previous example, however in this case the uneven distribution of the $\mathrm{DA}^{-}$caused the droplet to spin and pull in different directions which eventually lead to droplet splitting. An interesting application for this mechanism was cargo distribution. If cargo was placed within the initial droplet, upon splitting it was observed that the cargo was distributed evenly among the newly formed daughter droplets.

Lopez et al. [131] demonstrated that propulsion of a micro-boat could be achieved by incorporating an ionogel which was soaked in ethanol, into the boat design (Figure 24). When placed in water, the boat moved spontaneously in random directions. When the ionogel touched the water, ethanol molecules were expelled from the ionogel matrix at the rear of the boat and replaced with water molecules. The large difference in surface tension between water and ethanol caused an asymmetrical surface tension gradient between the end and the front of the boat, causing the boat to move in a forward motion. Lopez and co-workers explained that speed and direction of the boat was controlled by the size of the ionogel and stated that with larger gels speed increased while directional control decreased. Using smaller ionogels resulted in slower boat speeds with increased control over directional movement, probably due to release of less surfactant.

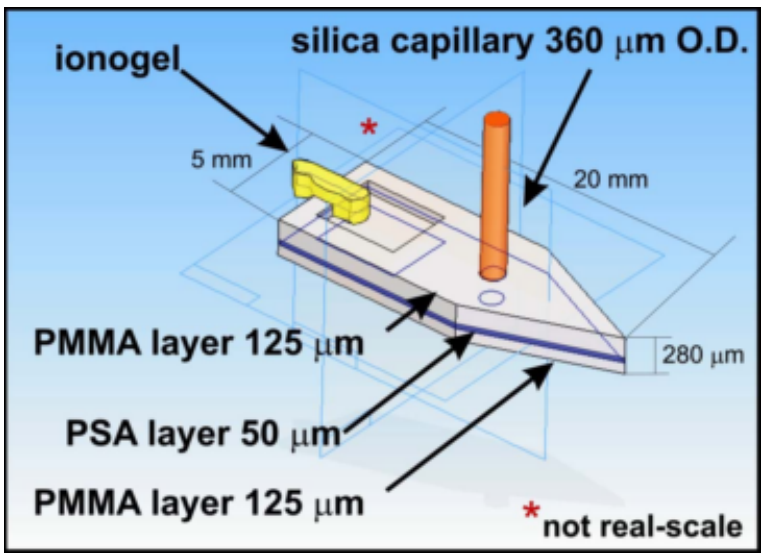

Figure 24. Schematic of micro-boat which contains the ethanol soaked ionogel.

Luo et al. [132] have demonstrated surfactant driven actuation by developing a micro-boat which contained a reservoir to hold the surfactant, namely isopropyl alcohol (IPA). When the boat was placed on water the surfactant left the boat and interacted with the water behind the boat, lowering the surface tension, and causing propulsion of the boat. The boat could travel up a $94.5 \mathrm{~cm}$ long channel with speeds of up to $10 \mathrm{~cm} \mathrm{~s}^{-1}$. The volume of surfactant (in the boat) and the volume of water (in the 
channel) both determined the speed of the boat as the propulsion force of the boat was dependent on the concentration of the IPA. During the experiments, water from the channel would enter the reservoir, thus reducing the concentration of the IPA, which in turn reduced the surface tension drop and lowered the speed of the boat.

The "toy boat" mechanism by which a small cardboard boat could be propelled on water by adding a small amount of surfactant (hand soap) to the rear of the boat [133], inspired this type of surfactant driven synthetic vehicle. However, this effect is short lived as the small amount of surfactant is quickly depleted and since the surfactant will remain at the surface of the water, the surface tension will no longer be modified by supplementary surfactant addition.

Sen et al. [123] described a novel method for vehicle movement in which the surfactant (needed for propulsion) was produced via a chemical reaction. These vehicles contained the polymer poly(2-ethyl cyanoacrylate) (PECA) which is an FDA approved polymer. When placed in a basic solution $(1 \mathrm{M} \mathrm{NaOH})$ PECA underwent a deesterification reaction, which resulted in rapid depolymerisation of PECA producing smaller molecules (ethanol) which were surface active (Figure 25).

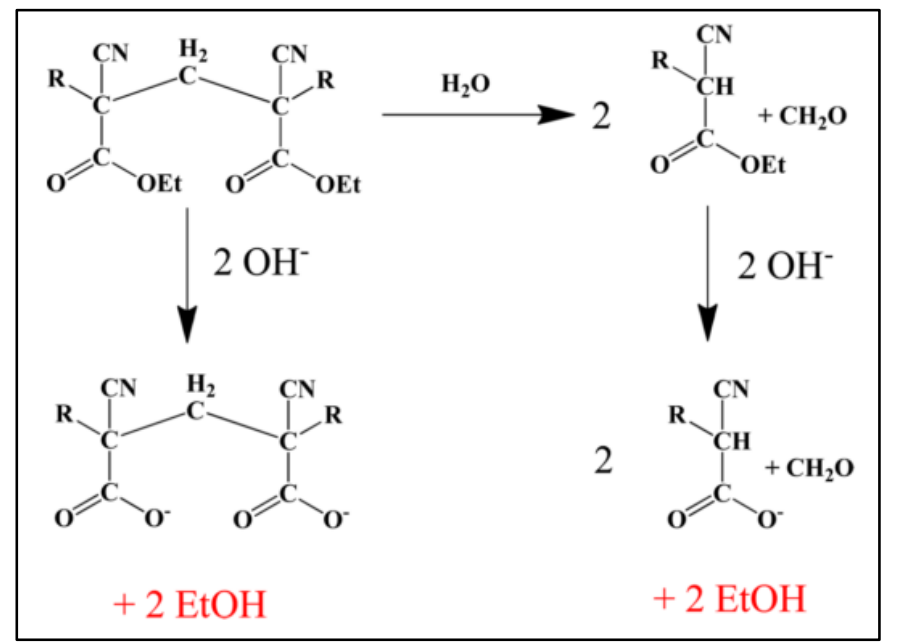

Figure 25. Mechanism for the depolymerisation of the polymer PECA. Reprinted with permission from ([123]). Copyright (2013) AmericanChemical Society.

Pipette tips were used as the vehicle in this study. One end of the tip was sealed with PDMS, which ensured that the chemical reaction could only occur at the open end of the pipette tip (Figure 26). The ethanol produced lowered the surface tension of the solution behind the pipette tip thus breaking the surface tension symmetry, which produced Marangoni like flows and propelled the vehicle forward (Figure 26). The vehicles in this study were capable of self-generating surface tension gradients while unidirectional movement was assured as the chemical reaction could only occur at one end of the tip. This type of "propulsion" mechanism also allowed for actuation in various media such as salt solutions and artificial serum. This was achieved by coating ion exchange beads with PECA; the ion exchange beads actively released $\mathrm{OH}^{-}$ions which triggered the depolymerisation of PECA, thus promoting vehicle "propulsion". The asymmetrical coating of the PECA again ensured that unidirectional movement was achieved. 


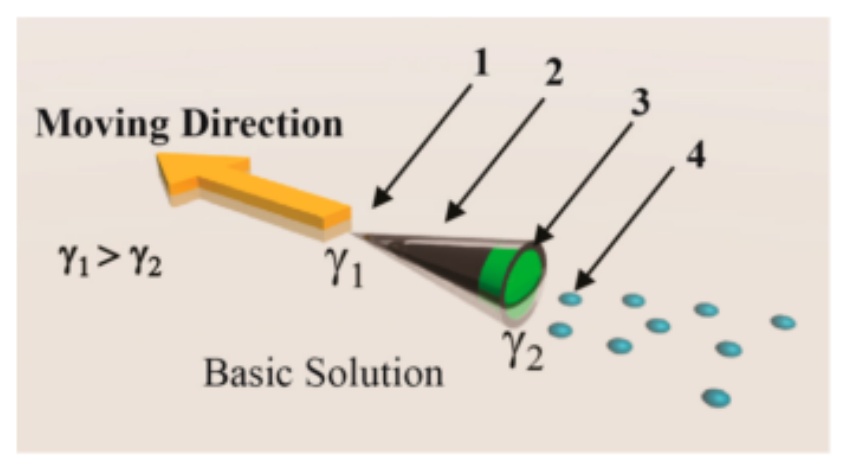

\section{1: Sealed End 2: Motor Body \\ 3: PECA Fuel 4: Depolymerization Products}

Figure 26. Schematic of actuation of PECA loaded vehicle. Reprinted with permission from ([123]). Copyright (2013) AmericanChemical Society.

Ban et al. [134] have shown the self-propelled motion of oil droplets at neutral $\mathrm{pH}$. The droplet used in this study contained the surfactant di(2-ethylhexyl) phosphoric acid (DEHPA). These droplets showed autonomous random movement due to Marangoni like flows created from the release of the DEHPA surfactant. As the $\mathrm{pH}$ of the aqueous solution was increased, the DEHPA was deprotonated, and its release form the droplet caused a decrease in the interfacial tension between the droplet and solution. This created circulating flow within the droplet. The motion of the droplet was maintained due to the cycle of deprotonated DEPHA being released into the aqueous phase and protonated DEPHA being supplied to the interface from within the droplet (Figure 27). Since the movement of the droplet was dependent on the deprotonation of the DEPHA surfactant, it was switched on and off by controlling the $\mathrm{pH}$ of the aqueous solution. Below $\mathrm{pH} 6$ the droplet did not move due to DEPHA not being deprotonated (and not being released). Above pH 6 the droplets would move spontaneously. However, the movement of the droplet was random and the direction was solely based on how the droplet was placed on top of the solution.

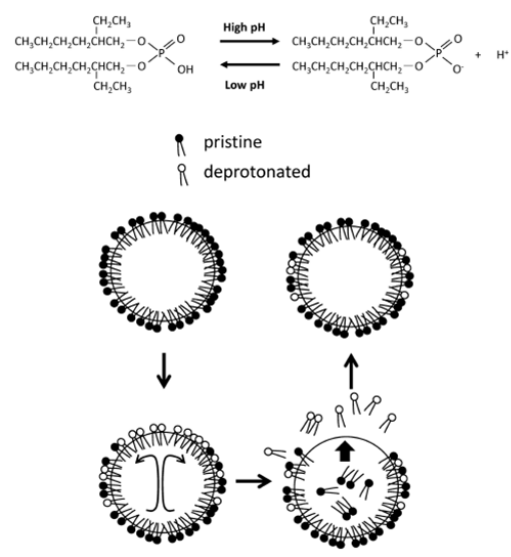

Figure 27. Structure of DEPHA surfactant and mechanism of movement of the oil droplet. Movement is due to the deprotonation of the DEPHA, which results in Marangoni convection within the droplet. This was maintained due to the replenishment of protonated DEPHA at the droplet/liquid interface. Reprinted with permission from ([134]). Copyright (2013) American Chemical Society.

Using the DEPHA surfactant, Ban et al. [135] demonstrated self-propelled droplets which moved in the direction of a higher concentration of heavy metal ions. In this study, Ban and coworkers again used an oil droplet which contained the DEPHA surfactant. However, two stimuli were used to control the movement of the droplet. Firstly, adjusting the $\mathrm{pH}$ to achieve droplet mobility and secondly creating metal ions gradients to move the droplets in a unidirectional fashion. 
The self-propelled motion of the droplets remained the same as in the previous example. However, the movement of the droplets towards higher concentrations of metal ions can be explained as follows; when the oil droplet was placed on a solution with a $\mathrm{pH}$ above 6 , the interface of the droplet was covered with negatively charged DEPHA molecules. These negatively charged molecules served as receptors for positively charged metal ions in the aqueous phase. When a gradient of metal ions was present in the solution, a DEHPA-metal complex was formed at the interface. When this complex was released into the aqueous solution, it created a surface tension gradient around the droplet. This caused Marangoni like flows which drove the droplet towards the area of highest concentration of metal ions. The speed of the droplet was controlled by adjusting the $\mathrm{pH}$ of the solution accordingly.

In a different approach, Florea et al. [136] demonstrated unique "photo-chemopropulsion" mechanism for droplet movement. The lipophilic droplets in this study were composed of the $\mathrm{pH}$ sensitive surfactants HDA and Chromoionophore I (CI) in DCM. Within the droplet, the weak acid HDA was deprotonated to $\mathrm{DA}^{-}$through interactions with the weak base $\mathrm{CI}$, which resulted in production of $\mathrm{CI}-\mathrm{H}^{+}$, a very efficient cationic surfactant. This surfactant associated with the $\mathrm{DA}^{-}$to form the salt $\left[\mathrm{CI}-\mathrm{H}^{+}\right]\left[\mathrm{DA}^{-}\right]$. The term photo-chemopropulsion was used as the release of the surfactant $\mathrm{CI}-\mathrm{H}^{+}$was controlled by altering the $\mathrm{pH}$ of the aqueous solution using a white light source. When the $\mathrm{pH}$ of the solution on which the droplet was resting was lowered below the $\mathrm{pK}_{\mathrm{b}}$ of the $\mathrm{DA}^{-}$, the CI- $\mathrm{H}^{+}$ surfactant was released from the droplet into the aqueous solution (Figure 28). Once released, the surfactant altered the surface tension symmetry around the droplet and created Marangoni like flows which drove the droplet away from the light source. The authors stated that the rate of the $\mathrm{pH}$ change can be controlled by the light intensity, producing droplet unidirectional movement with speeds up to $4000 \mu \mathrm{m} \mathrm{s}^{-1}$.

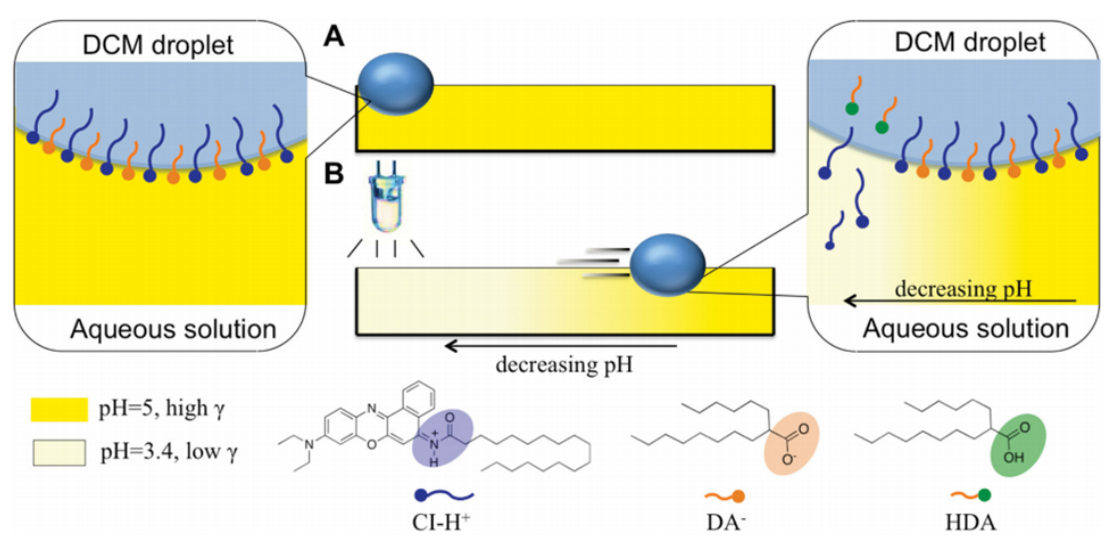

Figure 28. Photo-chemopropulsion of a micro droplet which is resting on an aqueous solution containing the photochromic molecule, spiropyran. A-depicts the droplet before white light irradiation and B-depicts the droplet after white light irradiation. After the introduction of the light source, the cationic surfactant $\mathrm{CI}-\mathrm{H}^{+}$is released, breaking the surface tension symmetry of the solution around the droplet and creating Marangoni like flows which drive the droplet away from the white light source. Reproduced from [136].

Florea and co-workers were able to control the $\mathrm{pH}$ of the aqueous solution by using an acidic photocromic molecule, namely a sulfonic acid spiropyran derivative ( $\left.\mathrm{SP}_{-} \mathrm{SO}_{3} \mathrm{H}\right)$. Dissolution of SP$\mathrm{SO}_{3} \mathrm{H}$ in the aqueous solution resulted in the dissociation of the sulfonic acid and the ring opening of the SP to form an equilibrium mixture of two merocyanine (MC) forms, protonated merocyanine $\left(\mathrm{MCH}^{+}-\mathrm{SO}_{3}{ }^{-}\right)$which is the predominant form, and deprotonated merocyanine $\left(\mathrm{MC}-\mathrm{SO}_{3}{ }^{-}\right)$. When the white light source was introduced, the $\mathrm{MC}_{-} \mathrm{SO}_{3}{ }^{-}$and $\mathrm{MCH}^{+}-\mathrm{SO}_{3}{ }^{-}$were converted back to the spiropyran sulfonate $\left(\mathrm{SP}_{-} \mathrm{SO}_{3}\right)$ form, releasing a proton $\left(\mathrm{H}^{+}\right)$in the process (Figure 29). This in turn caused the $\mathrm{pH}$ of the aqueous solution to drop from about $\mathrm{pH} 5$ to around $\mathrm{pH} 3.4$. Therefore, the authors were able to precisely control the $\mathrm{pH}$ (5.0 to 3.4) of a specific area of the solution, causing on demand surfactant release from the droplet to the aqueous solution. Using this method Florea and co- 
workers were able to achieve contactless control of the speed and directional movement of the droplet using only a white light source.

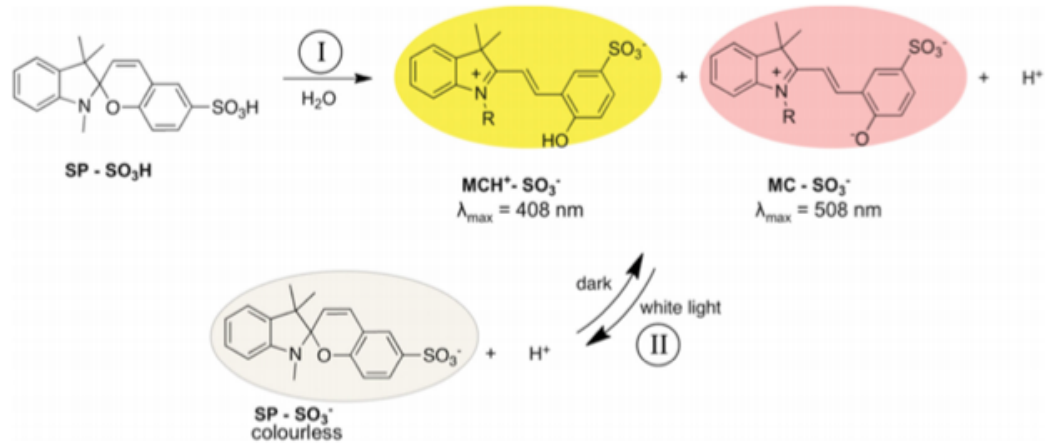

Figure 29. Structural change of the $\mathrm{SP}_{-} \mathrm{SO}_{3} \mathrm{H}$ photochromic molecule when dissolved in an aqueous solution under different illumination conditions. Reproduced from [136].

Another system in which the surfactant is contained within the "vehicle" is based on Ionic liquids. Francis et al [137] demonstrate the chemotactic movement of ionic liquid droplets, specifically droplets of trihexyl(tetradecyl)phosphonium chloride $\left(\left[\mathrm{P}_{6,6,6,14}\right][\mathrm{Cl}]\right)$. These ionic liquids were designed to move across the air/liquid interface and the motion was controlled by the triggered release of the $\left[\mathrm{P}_{6,6,6,14}\right]^{+}$, which is a very efficient cationic surfactant and a constituent of the IL droplet (Figure 30).

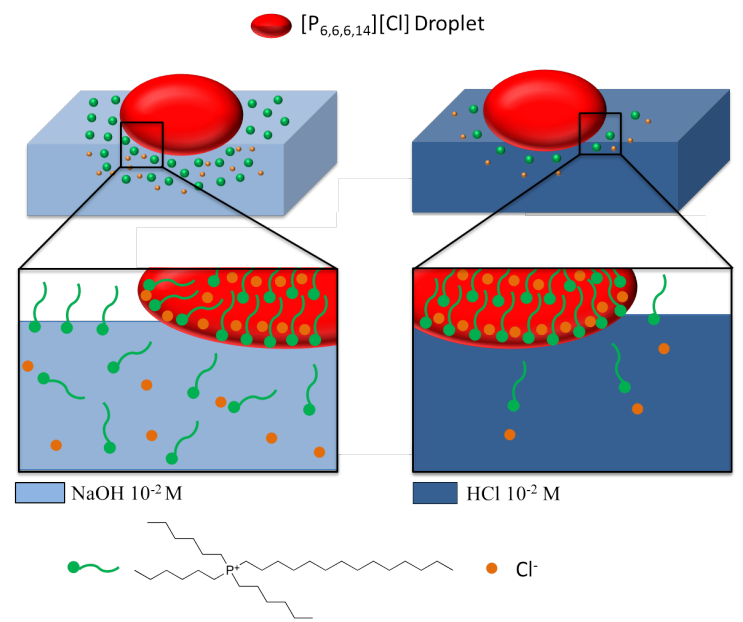

Figure 30. Diagram showing composition and relative solubility of $\left[\mathrm{P}_{6,6,6,14}\right][\mathrm{Cl}]$ in solutions of $10^{-2} \mathrm{M} \mathrm{NaOH}$ and $10^{-2} \mathrm{M} \mathrm{HCl}$. Reproduced from [137].

When the surfactant diffuses from the droplet into the aqueous phase it resulted in a drop of the local surface tension. The rate at which the surfactant was released depended on the solubility of the closely associated counter ion within the IL, in this case $\mathrm{Cl}^{-}$. Any transfer of the $\mathrm{Cl}^{-}$ion must be balanced by an equivalent transfer of $\left[\mathrm{P}_{6,6,6,14}\right]^{+}$in order to maintain overall charge neutrality within the droplet. The rate of release of $\mathrm{Cl}^{-}$was, in turn, dependent on the local aqueous $\mathrm{Cl}^{-}$concentration at the IL/aqueous boundary. Therefore when a droplet of $\left[\mathrm{P}_{6,6,6,14}\right][\mathrm{Cl}]$ was placed onto a aqueous solution, which had an imposed $\mathrm{Cl}^{-}$concentration gradient, there was a differential release of $\left[\mathrm{P}_{6,6,6,14}\right]^{+}$from the droplet into the aqueous solution. This resulted in the formation of an asymmetric surface tension gradient around the droplet, generating Marangoni like flows and driving the droplet from areas of low surface tension to high surface tension (Figure 31). 

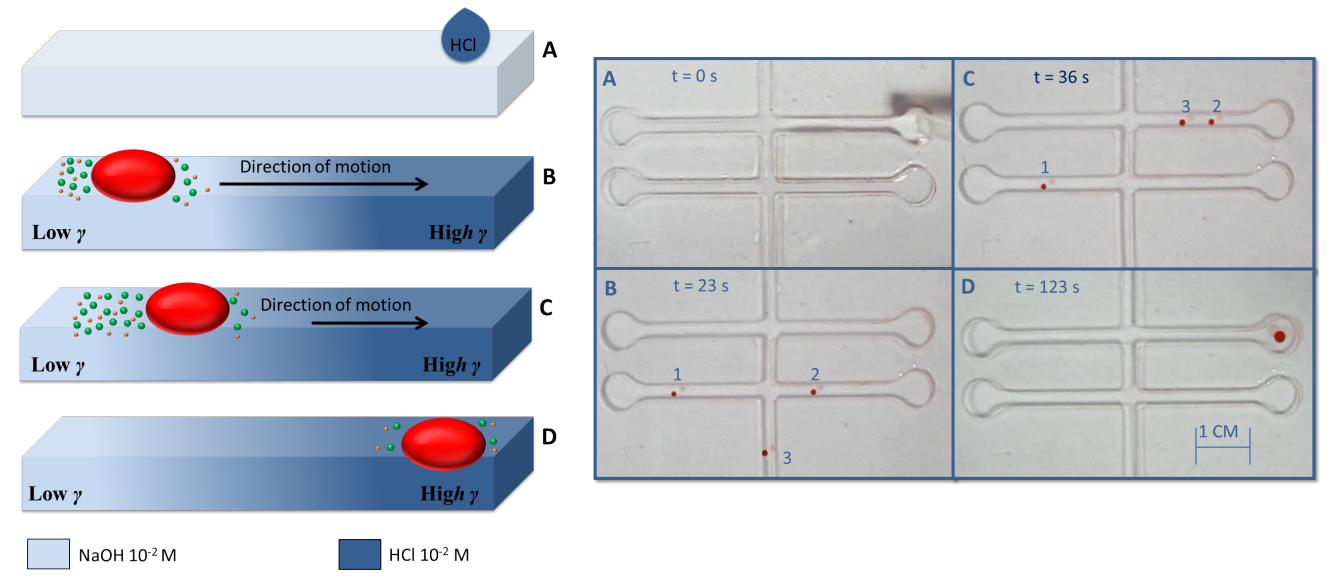

Figure 31. Schematic representation showing the chemotactic movement of Ionic liquid droplets in open fluidic channels (left) and sequence of video frames showing the chemotactic movement of multiple Ionic liquid droplets in an open fluidic channel (right). A-Depicts the creation of $\mathrm{Cl}^{-}$gradient, the channels were initially filled with a solution of $10^{-2} \mathrm{M} \mathrm{NaOH}$, at the desired destination a few drops of a $10^{-2} \mathrm{M}$ solution of $\mathrm{HCl}$ was placed. B-Shows the initial placement of the Ionic liquid droplet(s). C-The droplet(s) are propelled towards the highest area of surface tension. D - The droplet arrives at the desired destination. Reproduced from [137].

In this study Francis and coworkers demonstrated multiple ways to generate the required $\mathrm{Cl}^{-}$gradients, however the generated gradients are short lived and quickly come to equilibrium. This means the droplets can only be moved to a single destination for a limited period of time unless a mechanism for dynamic creation of local gradients is employed.

Francis et al. [138] have recently reported electrotactic ionic liquid droplets, in which the mechanism for movement of these droplets remains the same as the previous example, but the $\mathrm{Cl}^{-}$ gradients required for droplet movement are electrochemically generated using 3D printed electrodes embedded within the fluidic channels. In this paper, droplets of $\left[\mathrm{P}_{6,6,6,14}\right][\mathrm{Cl}]$ were electrotactically moved across the air/liquid interface of $10^{-3} \mathrm{M} \mathrm{NaCl}$ electrolyte solutions. When an external electric field was applied across an electrolyte solution, the mobile ions migrated towards their respective electrodes; i.e. anions towards the anode and cations towards the cathode, creating a $\mathrm{Cl}^{-}$concentration gradient along the channel. When a voltage was applied, a droplet of $\left[\mathrm{P}_{6,6,6,14}\right][\mathrm{Cl}]$ placed at the cathode asymmetrically released $\left[\mathrm{P}_{6,6,6,14}\right]^{+}$and autonomously moved towards the anode. Upon reversing the polarity of the electrodes, the gradient was also reversed and hence droplet movement could be reversed. In addition to this, the droplets could be steered into side channels at junctions, by polarizing appropriate electrode pairs (Figure 32). Electrotactic movement of the droplets allowed concentration gradients to be established and varied dynamically, and maintained for longer periods of time. This in turn enabled flexible control of microdroplet movement, introducing the ability to control the speed (depends on applied voltage), reversibility, and redirection into side channels. 


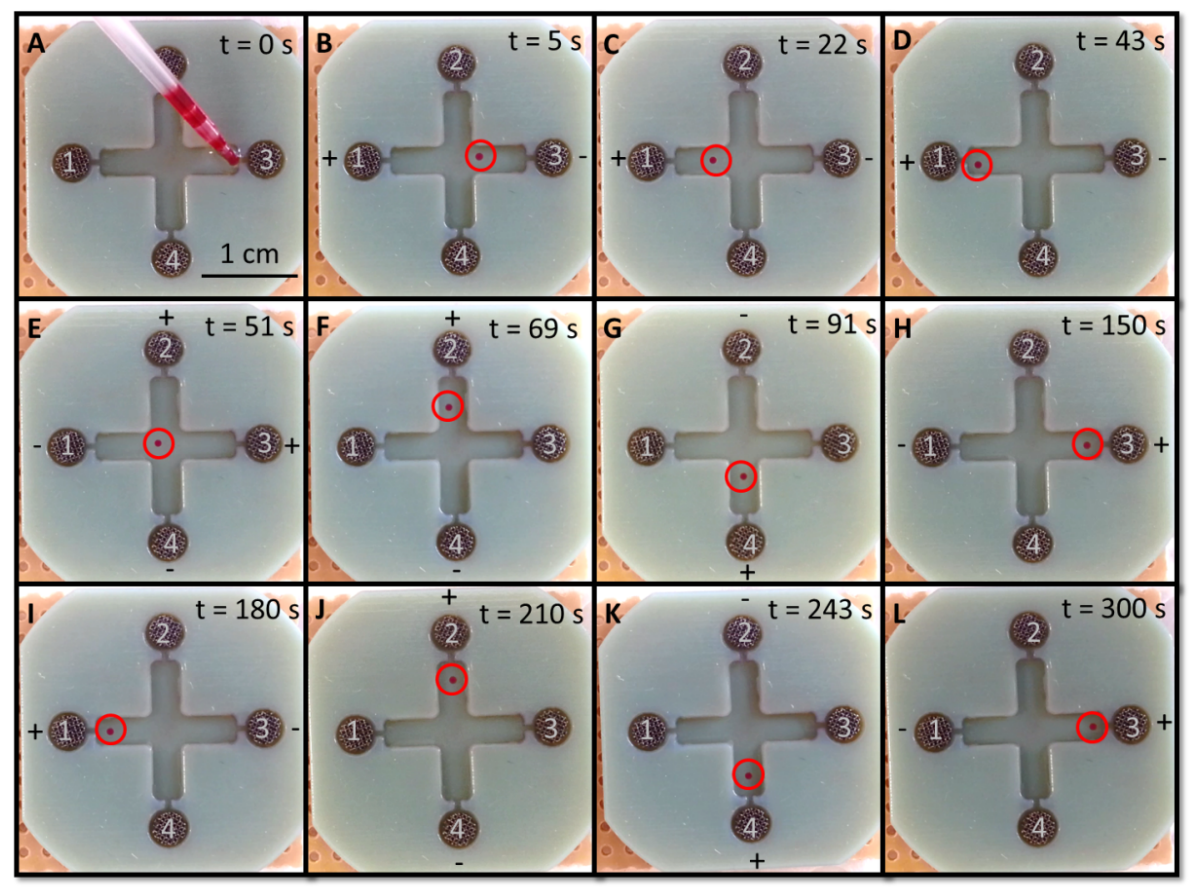

Figure 32. Sequence of snapshots showing the electrotactic movement of an Ionic liquid droplet. The channels are filled with a $10^{-3} \mathrm{M}$ solution of $\mathrm{NaCl}$ and a potential difference of $9 \mathrm{~V}$ is applied across selected electrodes $\mathrm{A}$ - The droplet is introduced $B-D$ The droplet is propelled from cathode (3) to anode (1). E-The polarity of electrodes (3) and (1) is reversed and the droplet begins to move to electrode (3). F-As the droplet approaches the junction, the potential difference is applied between electrodes (2) and (4) and removed from electrodes (3) and (1). The droplet then begins to migrate toward anode (2). $G$-Upon arriving at anode (2) the polarity of electrode (2) and (4) is reversed and the droplet moves towards the new anode (4). H-Upon arriving at anode (4) the potential is again reversed and using a similar method to sequence $F$ the droplet is returned to the starting position. I-L shows the process repeated in the same run. Reproduced from [138], (C) 2016 Elsevier B.V. All rights reserved.

Actuation of micro "vehicles" through localized changes in surface tension offers unique unidirectional control. However, there are still many challenges to face, such as moving the "vehicle" to defined specific areas within fluidic channels. Ideally, the vehicles should be controlled in a contactless manner, they should move spontaneously and require as little energy input as possible to achieve actuation. There is currently a movement towards tactic, functional and reactive droplets. This can be achieved by looking to nature for inspiration and many groups are reporting droplets which have biomimetic qualities, such as acting as vessels for chemical reactions [139], droplets with functional lipid bilayers [140, 141], droplets which can mimic DNA transcription and translation [142] and even droplets which show self-reproduction [143].

Li Sheng and Kensuke Kurihara [143], for example, report a novel vesicle-formation system. In this autocatalytic system octylaniline oil droplets act as a scaffold to produce vesicles by direct addition of a water-soluble catalytic aldehyde to form self-reproducing oil droplets. These droplets can then be transformed into giant vesicles via a further addition of a hydrophilic membrane precursor. In the study the catalytic aldehyde and octylaniline are combined together as an oil phase and dispersed in an aqueous solution as oil droplets. A condensation reaction between octylaniline and the catalytic aldehyde produces a catalytic imine, which further catalyzes the condensation reaction (Figure 33). Since this new catalytic imine is amphiphilic, its production resulted in more octylaniline being incorporated into the droplets from the aqueous phase, which in turn resulted in further reactions between the two precursors to produce more catalytic imine. 


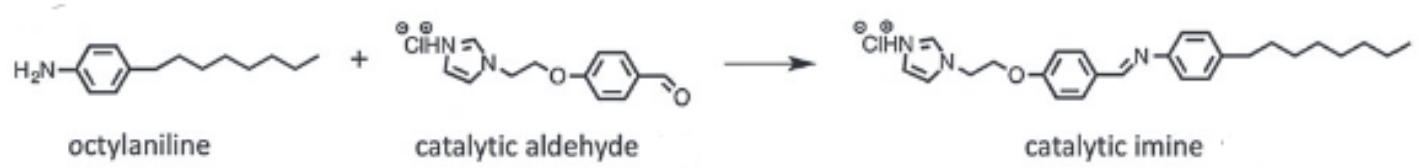

Figure 33. Condensation reaction between octylaniline and the catalytic aldehyde which produces a catalytic imine. Reproduced from [143] with permission from The Royal Society of Chemistry.

During the reaction the oil droplets began to increase in size until they reached a certain size threshold $(5-25 \mu \mathrm{m})$, at this point they began to divide to produce smaller droplets $(1-5 \mu \mathrm{m})$. This repeated growth and division indicated that in their system, the droplets maintained their composition and continued to produce the catalytic imine even after the division process (Figure 34).

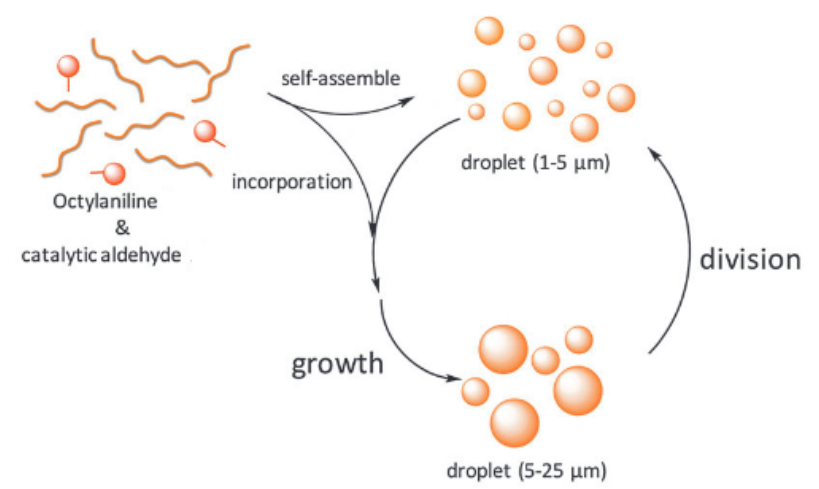

Figure 34. Schematic of the self-reproducing oil droplets. Reproduced from [143] with permission from The Royal Society of Chemistry.

Introduction of a hydrophilic membrane precursor into the system resulted in the formation of tubular giant vesicles (length of $50 \mu \mathrm{m}$ ). In the presence of the catalytic aldehyde, the octylaniline reacted with the hydrophilic membrane precursor to form vesicular membrane molecule (Figure 35).

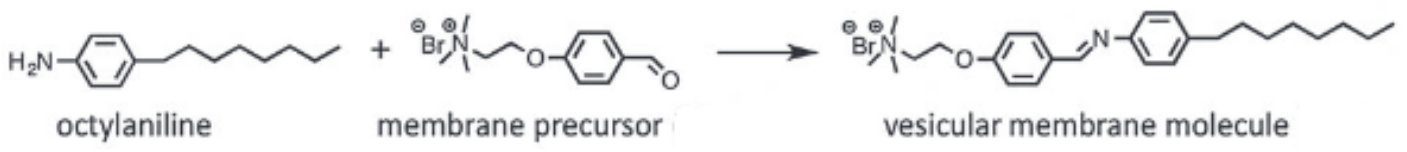

Figure 35. Reaction between octylaniline and the membrane precursor which produces the vesicular membrane molecule. Reproduced from [143] with permission from The Royal Society of Chemistry.

This reaction occurs initially in the aqueous phase, and according to Sheng this reaction may be also be capable of self-reproducing. The vesicular membrane molecules are then taken up by the oil droplets and slowly replace the octylaniline molecules. This replacement results in the transformation of the oil droplets into tubular and spherical giant vesicles. This study is of great interest as these droplets have begun to mimic primitive metabolic activity and also shows signs of early evolution of the droplets into larger more robust vesicles.

\section{Conclusions and Outlook}

We believe that the use of stimuli-responsive materials in microfluidics forms the core of autonomous microfluidic-based analytical devices, which are of considerably lower cost compared to their etched silicon (glass) counterparts, more biomimetic in nature and offer increased adaptability. Although this chapter is focused on the use of stimuli-responsive materials for fluid 
movement at the microscale, it gives the reader an insight into how the incorporation of such materials into microfluidics can allow for additional tasks beyond the transport of reagents or samples.

In living organisms, the fluidics system (e.g. cardiovascular system) performs multiple complex functions (e.g. sensing, detection, repair, waste removal; $\mathrm{pH}$ and temperature stabilization) in addition to the transport of fluids. Such characteristics of biological systems could be transferred into microfluidics through the creative use of adaptive materials and chemistries, to enable fundamental breakthroughs in chem/bio-sensing device performance. However, the future of biomimetic microfluidics relies on convincing demonstrators and their application in real scenarios where advanced functions such as autonomous fluid handling, sensing, detection and repair of damage, selfmanagement and healing, could be demonstrated.

\section{Acknowledgments}

This project has been funded by Science Foundation Ireland under the Insight initiative, grant SFI/12/RC/2289. LF and DD acknowledge COST Action MP1205 - 'Advances in Optofluidics: integration of optical control and photonics with microfluidics'. CD and DD acknowledge the European Union's Seventh Framework Programme for research, technological development, and demonstration, through the NAPES project grant agreement no. 604241.

\section{References and Notes}

[1] H.A. Stone, A.D. Stroock, A. Ajdari, Engineering Flows In Small Devices, Annual Review of Fluid Mechanics, 36(2004) 381-411.

[2] P. Gravesen, J. Branebjerg, Sonderga, O. rd Jensen, Microfluidics-a review, J Micromech Microeng J Mioromech Microeng, 3(1993) 16-82.

[3] D. Issadore, T. Franke, K.A. Brown, R.M. Westervelt, A microfluidic microprocessor: controlling biomimetic containers and cells using hybrid integrated circuit/microfluidic chips., Lab on a chip, 10(2010) 2937-43.

[4] D.J. Beebe, G.A. Mensing, G.M. Walker, Physics and Applications of Microfluidics in Biology, Annu Rev Biomed Eng, 4(2002) 261-86.

[5] M. He, A.E. Herr, Automated microfluidic protein immunoblotting, Nat Protocols, 5(2010) 1844-56.

[6] C. Plessy, L. Desbois, T. Fujii, P. Carninci, Population transcriptomics with single-cell resolution: A new field made possible by microfluidics, BioEssays, 35(2013) 131-40.

[7] S. Argentiere, G. Gigli, M.M. Irini Gerges, L. Blasi, Smart Microfluidics: The Role of Stimuli- Responsive Polymers in Microfluidic Devices, Advances in Microfluidics, InTech2012.

[8] S.K.Y. Tang, G.M. Whitesides, Basic Microfluidic and Soft Lithographic Techniques 2-1 Introduction 2-3 Materials for Fabricating Microfluidic Devices.

[9] D. Zhang, D. Zhang, L. Men, Q. Chen, Review Microfabrication and Applications of Opto-Microfluidic Sensors, (2011).

[10] F. Benito-Lopez, M. Antoñana-Díez, V.F. Curto, D. Diamond, Modular microfluidic valve structures based on reversible thermoresponsive ionogel actuators, Lab on a Chip, 14(2014) 3530.

[11] J. Ter Schiphorst, S. Coleman, J.E. Stumpel, A. Ben Azouz, D. Diamond, A.P.H.J. Schenning, Molecular Design of Light-Responsive Hydrogels, for in Situ Generation of Fast and Reversible Valves for Microfluidic Applications, Chemistry of Materials, 27(2015) 5925-31.

[12] J.B. Prettyman, D.T. Eddington, Leveraging stimuli responsive hydrogels for on/off control of mixing, Sensors \& Actuators: B Chemical, 157(2011) 722-6.

[13] D.J. Beebe, J.S. Moore, J.M. Bauer, Q. Yu, R.H. Liu, C. Devadoss, et al., Functional hydrogel structures for autonomous flow control inside microfluidic channels, Nature, 404(2000) 588-90.

[14] A. Richter, S. Klatt, G. Paschew, C. Klenke, Micropumps operated by swelling and shrinking of temperature-sensitive hydrogels., Lab on a chip, 9(2009) 613-8.

[15] L. Florea, A. Martin-Mayor, M.M. Bou-Ali, K. Meagher, D. Diamond, M. Tutar, et al., Adaptive coatings based on polyaniline for direct $2 \mathrm{D}$ observation of diffusion processes in microfluidic systems, Sensors and Actuators B: Chemical, 231(2016) 744-51. 
[16] R. Riahi, A. Tamayol, S.A.M. Shaegh, A.M. Ghaemmaghami, M.R. Dokmeci, A. Khademhosseini, Microfluidics for advanced drug delivery systems, Current Opinion in Chemical Engineering, 7(2015) 101-12.

[17] S.A. Pfeiffer, S. Nagl, Microfluidic platforms employing integrated fluorescent or luminescent chemical sensors: a review of methods, scope and applications, Methods and Applications in Fluorescence, 3(2015) 034003.

[18] D.T. Eddington, D.J. Beebe, Flow control with hydrogels, Advanced drug delivery reviews, 56(2004) 199210.

[19] D. Poelman, P.F. Smet, Methods for the determination of the optical constants of thin films from single transmission measurements: a critical review, Journal of Physics D: Applied Physics, 36(2003) 1850.

[20] E. Smela, N. Gadegaard, Surprising volume change in PPy (DBS): an atomic force microscopy study, Advanced Materials, 11(1999) 953-7.

[21] E. Smela, M. Kallenbach, J. Holdenried, Electrochemically driven polypyrrole bilayers for moving and positioning bulk micromachined silicon plates, Journal of microelectromechanical systems, 8(1999) 373-83.

[22] R. Baughman, Conducting polymer artificial muscles, Synthetic metals, 78(1996) 339-53.

[23] E. Smela, Conjugated polymer actuators for biomedical applications, Advanced materials, 15(2003) 48194.

[24] X.C. i Solvas, R.A. Lambert, L. Kulinsky, R.H. Rangel, M.J. Madou, Micromixing and flow manipulation with polymer microactuators, Microfluidics and nanofluidics, 11(2011) 405-16.

[25] J.D. Madden, R.A. Cush, T.S. Kanigan, I.W. Hunter, Fast contracting polypyrrole actuators, Synthetic Metals, 113(2000) 185-92.

[26] E.W. Jager, E. Smela, O. Inganäs, On-chip microelectrodes for electrochemistry with moveable PPy bilayer actuators as working electrodes, Sensors and Actuators B: Chemical, 56(1999) 73-8.

[27] E. Smela, Microfabrication of PPy microactuators and other conjugated polymer devices, Journal of micromechanics and microengineering, 9(1999) 1.

[28] E. Smela, A microfabricated movable electrochromic "pixel" based on polypyrrole, Advanced Materials, 11(1999) 1343-5.

[29] Y. Tanaka, T. Fujikawa, Y. Kazoe, T. Kitamori, An active valve incorporated into a microchip using a high strain electroactive polymer, Sensors and Actuators B: Chemical, 184(2013) 163-9.

[30] C. Murray, D. McCoul, E. Sollier, T. Ruggiero, X. Niu, Q. Pei, et al., Electro-adaptive microfluidics for active tuning of channel geometry using polymer actuators, Microfluidics and nanofluidics, 14(2013) 345-58.

[31] L. Dong, H. Jiang, Autonomous microfluidics with stimuli-responsive hydrogels, Soft matter, 3(2007) 1223-30.

[32] J. Moorthy, Hydrogels in microfluidics, SMART POLYMERS: Application in biotechnology and biomedicine, Taylor \& Francis, (2007) 437-57.

[33] E.J. Geiger, A.P. Pisano, F. Svec, A polymer-based microfluidic platform featuring on-chip actuated hydrogel valves for disposable applications, Journal of Microelectromechanical Systems, 19(2010) 944-50.

[34] G.M. Eichenbaum, P.F. Kiser, S.A. Simon, D. Needham, pH and ion-triggered volume response of anionic hydrogel microspheres, Macromolecules, 31(1998) 5084-93.

[35] S.K. De, N. Aluru, B. Johnson, W. Crone, D.J. Beebe, J. Moore, Equilibrium swelling and kinetics of pHresponsive hydrogels: Models, experiments, and simulations, Journal of Microelectromechanical Systems, 11(2002) 544-55.

[36] A. Kumar, A. Srivastava, I.Y. Galaev, B. Mattiasson, Smart polymers: physical forms and bioengineering applications, Progress in Polymer Science, 32(2007) 1205-37.

[37] S. Chaterji, I.K. Kwon, K. Park, Smart polymeric gels: redefining the limits of biomedical devices, Progress in polymer science, 32(2007) 1083-122.

[38] T. Miyata, T. Uragami, K. Nakamae, Biomolecule-sensitive hydrogels, Advanced drug delivery reviews, 54(2002) 79-98.

[39] E.D. Rodriguez, X. Luo, P.T. Mather, Linear/network poly ( $\varepsilon$-caprolactone) blends exhibiting shape memory assisted self-healing (SMASH), ACS applied materials \& interfaces, 3(2011) 152-61.

[40] T.R. Hoare, D.S. Kohane, Hydrogels in drug delivery: progress and challenges, Polymer, 49(2008) 19932007.

[41] P. Gupta, K. Vermani, S. Garg, Hydrogels: from controlled release to pH-responsive drug delivery, Drug discovery today, 7(2002) 569-79.

[42] E. Turan, T. Caykara, Swelling and network parameters of $\mathrm{pH}-$ sensitive poly (acrylamide-co-acrylic acid) hydrogels, Journal of applied polymer science, 106(2007) 2000-7. 
[43] D. Schmaljohann, Thermo-and pH-responsive polymers in drug delivery, Advanced drug delivery reviews, 58(2006) 1655-70.

[44] J. Chung, M. Yokoyama, M. Yamato, T. Aoyagi, Y. Sakurai, T. Okano, Thermo-responsive drug delivery from polymeric micelles constructed using block copolymers of poly ( $\mathrm{N}$-isopropylacrylamide) and poly (butylmethacrylate), Journal of Controlled Release, 62(1999) 115-27.

[45] H. Kanazawa, K. Yamamoto, Y. Matsushima, N. Takai, A. Kikuchi, Y. Sakurai, et al., Temperatureresponsive chromatography using poly ( $\mathrm{N}$-isopropylacrylamide)-modified silica, Analytical Chemistry, 68(1996) 100-5.

[46] G. Cirillo, T. Spataro, M. Curcio, U.G. Spizzirri, F.P. Nicoletta, N. Picci, et al., Tunable thermo-responsive hydrogels: synthesis, structural analysis and drug release studies, Materials Science and Engineering: C, 48(2015) 499-510.

[47] M. Prabaharan, J.F. Mano, Stimuli-responsive hydrogels based on polysaccharides incorporated with thermo-responsive polymers as novel biomaterials, Macromolecular bioscience, 6(2006) 991-1008.

[48] T. Okano, A. Kikuchi, Y. Sakurai, Y. Takei, N. Ogata, Temperature-responsive poly (Nisopropylacrylamide) as a modulator for alteration of hydrophilic/hydrophobic surface properties to control activation/inactivation of platelets, Journal of controlled release, 36(1995) 125-33.

[49] M. Heskins, J.E. Guillet, Solution properties of poly (N-isopropylacrylamide), Journal of Macromolecular Science-Chemistry, 2(1968) 1441-55.

[50] X. Wang, X. Qiu, C. Wu, Comparison of the coil-to-globule and the globule-to-coil transitions of a single poly (N-isopropylacrylamide) homopolymer chain in water, Macromolecules, 31(1998) 2972-6.

[51] J.-F. Lutz, A. Hoth, Preparation of ideal PEG analogues with a tunable thermosensitivity by controlled radical copolymerization of 2-(2-methoxyethoxy) ethyl methacrylate and oligo (ethylene glycol) methacrylate, Macromolecules, 39(2006) 893-6.

[52] J. Wang, Z. Chen, M. Mauk, K.-S. Hong, M. Li, S. Yang, et al., Self-actuated, thermo-responsive hydrogel valves for lab on a chip, Biomedical Microdevices, 7(2005) 313-22.

[53] A.K. Agarwal, L. Dong, D.J. Beebe, H. Jiang, Autonomously-triggered microfluidic cooling using thermoresponsive hydrogels, Lab on a Chip, 7(2007) 310-5.

[54] A. Dunne, C. Delaney, L. Florea, D. Diamond, Solvato-morphologically controlled, reversible NIPAAm hydrogel photoactuators, RSC Advances, 6(2016) 83296-302.

[55] L. Florea, D. Diamond, F. Benito-Lopez, Photo-Responsive Polymeric Structures Based on Spiropyran, Macromolecular Materials and Engineering, 297(2012) 1148-59.

[56] L. Florea, A. Hennart, D. Diamond, F. Benito-Lopez, Synthesis and characterisation of spiropyran-polymer brushes in micro-capillaries: Towards an integrated optical sensor for continuous flow analysis, Sensors and Actuators B: Chemical, 175(2012) 92-9.

[57] A. Tudor, L. Florea, D. Diamond, Multi-responsive semi-interpenetrating network hydrogels, (2015).

[58] A.M. Rosales, K.M. Mabry, E.M. Nehls, K.S. Anseth, Photoresponsive elastic properties of azobenzenecontaining poly (ethylene-glycol)-based hydrogels, Biomacromolecules, 16(2015) 798-806.

[59] E. Fischer, Y. Hirshberg, Formation of coloured forms of spirans by low-temperature irradiation, ROYAL SOC CHEMISTRY THOMAS GRAHAM HOUSE, SCIENCE PARK, MILTON RD, CAMBRIDGE CB4 0WF, CAMBS, ENGLAND1952, pp. 4522-4.

[60] K. Sumaru, T. Takagi, T. Satoh, T. Kanamori, Photo-induced reversible proton dissociation of spirobenzopyran in aqueous systems, Journal of Photochemistry and Photobiology A: Chemistry, 261(2013) 4652.

[61] Y. Wang, N. Ma, Z. Wang, X. Zhang, Photocontrolled reversible supramolecular assemblies of an azobenzene-containing surfactant with $\alpha$-cyclodextrin, Angewandte Chemie International Edition, 46(2007) 2823-6.

[62] G.S. Kumar, D. Neckers, Photochemistry of azobenzene-containing polymers, Chemical Reviews, 89(1989) 1915-25.

[63] K. Matsuda, M. Irie, Diarylethene as a photoswitching unit, Journal of Photochemistry and Photobiology C: Photochemistry Reviews, 5(2004) 169-82.

[64] J.E. Stumpel, B. Ziółkowski, L. Florea, D. Diamond, D.J. Broer, A.P. Schenning, Photoswitchable ratchet surface topographies based on self-protonating spiropyran-NIPAAM hydrogels, ACS applied materials \& interfaces, 6(2014) 7268-74. 
[65] L. Florea, D. Diamond, F. Benito-Lopez, Opto-Smart Systems in Microfluidics, Research Perspectives on Functional Micro-and Nanoscale Coatings, (2016) 265.

[66] B. Ziółkowski, L. Florea, J. Theobald, F. Benito-Lopez, D. Diamond, Self-protonating spiropyran-coNIPAM-co-acrylic acid hydrogel photoactuators, Soft Matter, 9(2013) 8754-60.

[67] J. ter Schiphorst, S. Coleman, J.E. Stumpel, A. Ben Azouz, D. Diamond, A.P. Schenning, Molecular design of light-responsive hydrogels, for in situ generation of fast and reversible valves for microfluidic applications, Chemistry of Materials, 27(2015) 5925-31.

[68] S. Sugiura, A. Szilágyi, K. Sumaru, K. Hattori, T. Takagi, G. Filipcsei, et al., On-demand microfluidic control by micropatterned light irradiation of a photoresponsive hydrogel sheet, Lab on a Chip, 9(2009) 196-8.

[69] S. Sugiura, K. Sumaru, K. Ohi, K. Hiroki, T. Takagi, T. Kanamori, Photoresponsive polymer gel microvalves controlled by local light irradiation, Sensors and Actuators A: Physical, 140(2007) 176-84.

[70] K. Sumaru, T. Takagi, S. Sugiura, T. Kanamori, Spiropyran-Functionalized Hydrogels, Soft Actuators, Springer2014, pp. 219-29.

[71] A. Szilágyi, K. Sumaru, S. Sugiura, T. Takagi, T. Shinbo, M. Zrínyi, et al., Rewritable microrelief formation on photoresponsive hydrogel layers, Chemistry of materials, 19(2007) 2730-2.

[72] K. Sumaru, K. Ohi, T. Takagi, T. Kanamori, T. Shinbo, Photoresponsive properties of poly (Nisopropylacrylamide) hydrogel partly modified with spirobenzopyran, Langmuir, 22(2006) 4353-6.

[73] F. Benito-Lopez, R. Byrne, A.M. Răduţă, N.E. Vrana, G. McGuinness, D. Diamond, Ionogel-based lightactuated valves for controlling liquid flow in micro-fluidic manifolds, Lab on a Chip, 10(2010) 195-201.

[74] M. Zrinyi, Intelligent polymer gels controlled by magnetic fields, Colloid and Polymer Science, 278(2000) 98-103.

[75] N.S. Satarkar, W. Zhang, R.E. Eitel, J.Z. Hilt, Magnetic hydrogel nanocomposites as remote controlled microfluidic valves, Lab on a Chip, 9(2009) 1773-9.

[76] S. Ghosh, C. Yang, T. Cai, Z. Hu, A. Neogi, Oscillating magnetic field-actuated microvalves for micro-and nanofluidics, Journal of Physics D: Applied Physics, 42(2009) 135501.

[77] N. Hohlbein, A. Shaaban, A. Schmidt, Remote-controlled activation of self-healing behavior in magnetoresponsive ionomeric composites, Polymer, 69(2015) 301-9.

[78] X. i Solvas, Droplet microfluidics: recent developments and future applications, Chem Commun, 47(2011) $1936-42$.

[79] S.-Y. Teh, R. Lin, L.-H. Hung, A. Lee, Droplet microfluidics, Lab on a chip, 8(2008) 198-220.

[80] D. Weibel, G. Whitesides, Applications of microfluidics in chemical biology, Curr Opin Chem Biol, 10(2006) 584-91.

[81] G.M. Whitesides, The origins and the future of microfluidics, Nature, 442(2006) 368-73.

[82] D. Baigl, Photo-actuation of liquids for light-driven microfluidics: state of the art and perspectives, Lab on a chip, 12(2012) 3637-53.

[83] S.-Y. Park, S. Kalim, C. Callahan, M. Teitell, E. Chiou, A light-induced dielectrophoretic droplet manipulation platform, Lab on a chip, 9(2009) 3228-35.

[84] C. Pei Yu, M. Hyejin, T. Hiroshi, K. Chang-Jin, C.W. Ming, Light actuation of liquid by optoelectrowetting, Sensors and Actuators A: Physical, 104(2003) 222-8.

[85] C. Han-Sheng, K. Aloke, T.W. Steven, Open optoelectrowetting droplet actuation, Appl Phys Lett, 93(2008) 9064104.

[86] S.N. Pei, J.K. Valley, S.L. Neale, A. Jamshidi, H.-Y. Hsu, M.C. Wu, Light-actuated digital microfluidics for large-scale, parallel manipulation of arbitrarily sized droplets, Micro Electro Mechanical Systems (MEMS), 2010 IEEE 23rd International Conference on, IEEE2010, pp. 252-5.

[87] S. Wang, Y. Song, L. Jiang, Photoresponsive surfaces with controllable wettability, Journal of Photochemistry and Photobiology C: Photochemistry Reviews, 8(2007) 18-29.

[88] S. Chaeyeon, K. Kipom, L. Kyuyong, P. Hyuk Kyu, Thermochemical control of oil droplet motion on a solid substrate, Appl Phys Lett, 93(2008) 084102.

[89] P. Vikram, M. Nadjoua, R.S. Subramanian, Thermocapillary Motion of a Liquid Drop on a Horizontal Solid Surface, Langmuir, 24(2008).

[90] J. Zhenjun, H. Xiaoyang, N. Nam-Trung, A. Patrick, Thermocapillary actuation of droplet in a planar microchannel, Microfluid Nanofluid, 5(2007) 205-14.

[91] A. Darhuber, J. Valentino, S. Troian, Planar digital nanoliter dispensing system based on thermocapillary actuation, Lab on a chip, 10(2010) 1061-71.

[92] J. Gomba, G. Homsy, Regimes of thermocapillary migration of droplets under partial wetting conditions, J Fluid Mech, 647(2010) 125-42. 
[93] A.A. Darhuber, J.P. Valentino, S.M. Troian, S. Wagner, Thermocapillary actuation of droplets on chemically patterned surfaces by programmable microheater arrays, Journal of Microelectromechanical Systems, 12(2003) 873-9.

[94] E. Bormashenko, R. Pogreb, Y. Bormashenko, A. Musin, T. Stein, New investigations on ferrofluidics: ferrofluidic marbles and magnetic-field-driven drops on superhydrophobic surfaces, Langmuir : the ACS journal of surfaces and colloids, 24(2008) 12119-22.

[95] A. Rida, M. Gijs, Manipulation of self-assembled structures of magnetic beads for microfluidic mixing and assaying, Anal Chem, 76(2004) 6239-46.

[96] Y. Zhao, J. Fang, H. Wang, X. Wang, T. Lin, Magnetic liquid marbles: manipulation of liquid droplets using highly hydrophobic Fe3O4 nanoparticles, Advanced materials (Deerfield Beach, Fla), 22(2010) 707-10.

[97] C.-Y. Wen, C.-P. Yeh, C.-H. Tsai, L.-M. Fu, Rapid magnetic microfluidic mixer utilizing AC electromagnetic field, Electrophoresis, 30(2009) 4179-86.

[98] Y. Wang, Y. Zhao, S.K. Cho, Efficient in-droplet separation of magnetic particles for digital microfluidics, Journal of Micromechanics and Microengineering, 17(2007) 2148.

[99] Y. Zhao, J. Fang, H. Wang, X. Wang, T. Lin, Magnetic liquid marbles: manipulation of liquid droplets using highly hydrophobic Fe3O4 nanoparticles, Adv Mater, 22(2010) 707-10.

[100] K. Choi, A. Ng, R. Fobel, A. Wheeler, Digital microfluidics, Annu Rev Anal Chem (Palo Alto Calif), 5(2012) 413-40.

[101] R.B. Fair, Digital microfluidics: is a true lab-on-a-chip possible?, Microfluid Nanofluid, 3(2007) 245-81.

[102] S. Vijay, K.P. Vamsee, B.F. Richard, An integrated digital microfluidic lab-on-a-chip for clinical diagnostics on human physiological fluidsThe Science and Application of Droplets in Microfluidic Devices., Lab on a Chip, 4(2004) 310-5.

[103] H. Zhishan, L.R. Jeremy, E.E. Allen, S. Vijay, K.P. Vamsee, A.S. Wiley, et al., Multiplexed Real-Time Polymerase Chain Reaction on a Digital Microfluidic Platform, Anal Chem, 82(2010) 2310-6.

[104] Y.-H. Chang, G.-B. Lee, F.-C. Huang, Y.-Y. Chen, J.-L. Lin, Integrated polymerase chain reaction chips utilizing digital microfluidics, Biomed Microdevices, 8(2006) 215-25.

[105] M. Abdelgawad, M. Watson, A. Wheeler, Hybrid microfluidics: a digital-to-channel interface for in-line sample processing and chemical separations, Lab on a chip, 9(2009) 1046-51.

[106] I. Barbulovic-Nad, H. Yang, P. Park, A. Wheeler, Digital microfluidics for cell-based assays, Lab on a chip, 8(2008) 519-26.

[107] V. Luk, A. Wheeler, A digital microfluidic approach to proteomic sample processing, Anal Chem, 81(2009) 4524-30.

[108] P.Y. Paik, V.K. Pamula, K. Chakrabarty, Adaptive cooling of integrated circuits using digital microfluidics, Very Large Scale Integration (VLSI) Systems, IEEE Transactions on, 16(2008) 432-43.

[109] S.W. Walker, B. Shapiro, Modeling the Fluid Dynamics of Electrowetting on Dielectric (EWOD), Journal of Microelectromechanical Systems, 15(2006) 986-1000.

[110] F. Saeki, J. Baum, H. Moon, J.-Y. Yoon, C. Kim, R. Garrell, Electrowetting on dielectrics (EWOD): reducing voltage requirements for microfluidics, Polym Mater Sci Eng, 85(2001) 12-3.

[111] J. Gong, C.-J.C. Kim, All-electronic droplet generation on-chip with real-time feedback control for EWOD digital microfluidics, Lab on a chip, 8(2008) 898-906.

[112] L. Malic, D. Brassard, T. Veres, M. Tabrizian, Integration and detection of biochemical assays in digital microfluidic LOC devices, Lab on a chip, 10(2010) 418-31.

[113] L. Wang, L. Flanagan, E. Monuki, N. Jeon, A. Lee, Dielectrophoresis switching with vertical sidewall electrodes for microfluidic flow cytometry, Lab on a chip, 7(2007) 1114-20.

[114] Y. Li, C. Dalton, H. Crabtree, G. Nilsson, K. Kaler, Continuous dielectrophoretic cell separation microfluidic device, Lab on a chip, 7(2007) 239-48.

[115] E. Cummings, Streaming dielectrophoresis for continuous-flow microfluidic devices, IEEE engineering in medicine and biology magazine : the quarterly magazine of the Engineering in Medicine \& Biology Society, 22(2003) 75-84.

[116] R. Rohit, G. Devens, A.G. Antonio, H. Mark, J.L. Taraci, T. Clement, et al., Lotus Effect Amplifies LightInduced Contact Angle Switching, The Journal of Physical Chemistry B, 108(2004) 12640-2.

[117] L. Siewierski, W. Brittain, S. Petrash, M. Foster, Photoresponsive monolayers containing in-chain azobenzene, Langmuir, 12(1996) 5838-44.

[118] S.-K. Oh, M. Nakagawa, K. Ichimura, Photocontrol of liquid motion on an azobenzene monolayer, J Mater Chem, 12(2002) 2262-9. 
[119] P. Vikram, M. Nadjoua, R.S. Subramanian, Thermocapillary Motion of a Liquid Drop on a Horizontal Solid Surface, Langmuir, 24(2008) 5185-93.

[120] A. Wixforth, C. Strobl, C. Gauer, A. Toegl, J. Scriba, Z. v Guttenberg, Acoustic manipulation of small droplets, Anal Bioanal Chem, 379(2004) 982-91.

[121] Z. Guttenberg, H. Müller, H. Habermüller, A. Geisbauer, J. Pipper, J. Felbel, et al., Planar chip device for PCR and hybridization with surface acoustic wave pump, Lab on a Chip, 5(2005) 308-17.

[122] B. Paul, P.B. Craig, E. Julian, Stimuli-responsive surfactants, Soft Matter, 9(2013) 2365-74.

[123] H. Zhang, W. Duan, L. Liu, A. Sen, Depolymerization-powered autonomous motors using biocompatible fuel, J Am Chem Soc, 135(2013) 15734-7.

[124] P. Brown, C.P. Butts, J. Eastoe, Stimuli-responsive surfactants, Soft Matter, 9(2013) 2365-74.

[125] A. Diguet, H. Li, N. Queyriaux, Y. Chen, D. Baigl, Photoreversible fragmentation of a liquid interface for micro-droplet generation by light actuation, Lab on a chip, 11(2011) 2666-9.

[126] A. Diguet, R.-M. Guillermic, N. Magome, A. Saint-Jalmes, Y. Chen, K. Yoshikawa, et al., Photomanipulation of a droplet by the chromocapillary effect, Angewandte Chemie (International ed in English), 48(2009) 9281-4.

[127] T. Toyota, N. Maru, M. Hanczyc, T. Ikegami, T. Sugawara, Self-propelled oil droplets consuming "fuel" surfactant, J Am Chem Soc, 131(2009) 5012-3.

[128] J. Cejkova, M. Novak, F. Stepanek, M.M. Hanczyc, Dynamics of chemotactic droplets in salt concentration gradients, Langmuir, 30(2014) 11937-44.

[129] I. Lagzi, S. Soh, P. Wesson, K. Browne, B. Grzybowski, Maze solving by chemotactic droplets, J Am Chem Soc, 132(2010) 1198-9.

[130] K. Browne, D. Walker, K. Bishop, B. Grzybowski, Self-division of macroscopic droplets: partitioning of nanosized cargo into nanoscale micelles, Angewandte Chemie (International ed in English), 49(2010) 6756-9.

[131] R. Byrne, F. Lopez, S. Scaramagnani, M. Higgins, G.G. Wallace, D. Diamond, Beads, boats and switches: making things happen with molecular photoswitches, IEEE, (2009) 139-43.

[132] C. Luo, H. Li, X. Liu, Propulsion of microboats using isopropyl alcohol as a propellant, Journal of Micromechanics and Microengineering, 18(2008) 067002.

[133] R. Charles, B. Ashley, M. Tiddo Jonathan, Easy Demonstration of the Marangoni Effect by Prolonged and Directional Motion: "Soap Boat 2.0", J Chem Educ, 90(2013) 1353-7.

[134] T. Ban, T. Yamagami, H. Nakata, Y. Okano, pH-Dependent Motion of Self-Propelled Droplets due to Marangoni Effect at Neutral pH, Langmuir, 29(2013) 2554-61.

[135] T. Ban, K. Tani, H. Nakata, Y. Okano, Self-propelled droplets for extracting rare-earth metal ions, Soft matter, 10(2014) 6316-20.

[136] L. Florea, K. Wagner, P. Wagner, G.G. Wallace, F. Benito-Lopez, D.L. Officer, et al., PhotoChemopropulsion-Light-Stimulated Movement of Microdroplets, Adv Mater, 26(2014) 7339-45.

[137] W. Francis, C. Fay, L. Florea, D. Diamond, Self-propelled chemotactic ionic liquid droplets, Chem Commun, 51(2015) $2342-4$.

[138] W. Francis, K. Wagner, S. Beirne, D.L. Officer, G.G. Wallace, L. Florea, et al., Electrotactic ionic liquid droplets, Sensors Actuators B: Chem, 239(2017) 1069-75.

[139] R.M. Bain, C.J. Pulliam, F. Thery, R.G. Cooks, Accelerated Chemical Reactions and Organic Synthesis in Leidenfrost Droplets, Angew Chem, (2016).

[140] K. Funakoshi, H. Suzuki, S. Takeuchi, Lipid bilayer formation by contacting monolayers in a microfluidic device for membrane protein analysis, Anal Chem, 78(2006) 8169-74.

[141] M.A. Holden, D. Needham, H. Bayley, Functional bionetworks from nanoliter water droplets, J Am Chem Soc, 129(2007) 8650-5.

[142] P. Torre, C.D. Keating, S.S. Mansy, Multiphase Water-in-Oil Emulsion Droplets for Cell-Free Transcription-Translation, Langmuir, 30(2014) 5695-9.

[143] L. Sheng, K. Kurihara, Transformation of oil droplets into giant vesicles, Chem Commun, (2016). 